\title{
Spectrum of quantum transfer matrices via classical many-body systems
}

\author{
A. Gorsky, ${ }^{a, b}$ A. Zabrodin ${ }^{a, b, c, d}$ and A. Zotov ${ }^{a, b, e}$ \\ a ITEP, \\ Bolshaya Cheremushkinskaya str. 25, 117218, Moscow, Russia \\ ${ }^{b}$ MIPT, \\ Inststitutskii per. 9, 141700, Dolgoprudny, Moscow region, Russia \\ ${ }^{c}$ Institute of Biochemical Physics, \\ Kosygina str. 4, 119991, Moscow, Russia \\ ${ }^{d}$ National Research University Higher School of Economics, \\ Myasnitskaya str. 20, 101000, Moscow, Russia \\ e Steklov Mathematical Institute, RAS, \\ Gubkina str. 8, 119991, Moscow, Russia \\ E-mail: gorsky@itep.ru, zabrodin@itep.ru, zotov@mi.ras.ru
}

ABSTRACT: In this paper we clarify the relationship between inhomogeneous quantum spin chains and classical integrable many-body systems. It provides an alternative (to the nested Bethe ansatz) method for computation of spectra of the spin chains. Namely, the spectrum of the quantum transfer matrix for the inhomogeneous $\mathfrak{g l}_{n}$-invariant XXX spin chain on $N$ sites with twisted boundary conditions can be found in terms of velocities of particles in the rational $N$-body Ruijsenaars-Schneider model. The possible values of the velocities are to be found from intersection points of two Lagrangian submanifolds in the phase space of the classical model. One of them is the Lagrangian hyperplane corresponding to fixed coordinates of all $N$ particles and the other one is an $N$-dimensional Lagrangian submanifold obtained by fixing levels of $N$ classical Hamiltonians in involution. The latter are determined by eigenvalues of the twist matrix. To support this picture, we give a direct proof that the eigenvalues of the Lax matrix for the classical Ruijsenaars-Schneider model, where velocities of particles are substituted by eigenvalues of the spin chain Hamiltonians, calculated through the Bethe equations, coincide with eigenvalues of the twist matrix, with certain multiplicities. We also prove a similar statement for the $\mathfrak{g l}_{n}$ Gaudin model with $N$ marked points (on the quantum side) and the Calogero-Moser system with $N$ particles (on the classical side). The realization of the results obtained in terms of branes and supersymmetric gauge theories is also discussed.

Keywords: Duality in Gauge Field Theories, Integrable Hierarchies, Bethe Ansatz

ArXiv EPRINT: 1310.6958 


\section{Contents}

1 Introduction 1

2 Classical integrable many-body systems 4

3 Quantum spin chains and Gaudin models $\quad 6$

$\begin{array}{lll}4 & \text { The QC duality } & 9\end{array}$

5 Relation to branes and gauge theories $\quad 14$

$\begin{array}{llr}6 & \text { Discussion } & 16\end{array}$

$\begin{array}{lr}\text { A Proofs of propositions } & 18\end{array}$

\section{Introduction}

The notion of duality was introduced into the landscape of integrable models long ago. In a general sense, it connects integrable systems of different types and ranges from purely quantum field theory models like sin-Gordon and Thirring to purely classical integrable systems with finite number of degrees of freedom. On the other hand, dualities play a key role in supersymmetric gauge theories and their stringy and M-theory UV completions. In this context, we again have plenty of correspondences between the gauge theories like S-duality, T-duality, mirror symmetry, Seiberg duality etc. All of them have one or another geometrical origin and many of them can be most clearly expressed in terms of brane motion.

It is known nowadays that integrable models are closely related to the SUSY gauge theories in different dimensions and provide an effective tool to describe them in the lowenergy sector in the spirit of the Seiberg-Witten solution $[1,2]$. In particular, some lowenergy effective actions in $\mathrm{N}=2$ gauge theories have been obtained via mapping to the corresponding integrable many-body systems [3,4] (see [5] for a review). To some extent the integrable systems capture the emerging hidden symmetry when the integration over the moduli space of the non-perturbative solutions relevant for the particular SUSY gauge theory is taken into account. The degrees of freedom of the corresponding integrable systems as well as the commuting flows are identified with the coordinates of the different branes localized in different dimensions in ten- or eleven- dimensional geometry of the string and M-theory.

The relation between gauge theories and integrable systems can be used in both directions. The Adams-Harnard-Hurtubise $(\mathrm{AHH})$ duality $[6,7]$ together with the results of $[8,9]$ allows us to obtain an interesting interrelation [10] between Heisenberg magnetic 
chains and their degenerate cases known as Gaudin models [11, 12]. It was known for quite a long time that a similar phenomenon takes place in the theory of classical many-body systems of the Calogero-Moser (CM) [13-16] or the Ruijsenaars-Schneider (RS) [17, 18] types. These issues have been discussed in [19-22]. Recently, an improved version of the AHH duality (the spectral duality) turned out to be a very effective tool in analyzing the 2d/4d duality [23-26] and the AGT correspondence [27-34]. The AHH duality has been identified as the $3 \mathrm{~d}$ mirror symmetry [35].

In this paper we will focus on the intriguing classical-quantum $(Q C)$ duality between integrable models with finite number of degrees of freedom. One of the models is quantum and another one is classical. Let us stress that this correspondence (based on the recent results of [36-41] and older results of [42]) has nothing to do with the quasiclassical limit. Presumably, it exists for integrable models only.

This kind of duality suggests an alternative way for calculating joint spectra of commuting quantum operators (transfer matrices and Hamiltonians), without any use of the coordinate or algebraic Bethe ansatz technique [43-48], which so far was a key tool in any exact solution of quantum integrable models with non-trivial interaction. There is also no need in such an unavoidable intermediate step as solving Bethe equations. The spectra of quantum Hamiltonians of an integrable system appear to be encoded in algebraic properties of the Lax matrix for a very different and purely classical model!

In a nutshell, the quantum spectral problem for an integrable spin chain on $N$ sites reformulated in terms of the "QC-dual" $N$-body integrable 1D systems of classical mechanics is as follows. Let us fix coordinates $q_{i}$ of the $N$ classical particles and levels of the $N$ Hamiltonians $H_{i}$ in involution. Then possible values of particles velocities give spectra of the spin chain Hamiltonians. In other words, one may say that the eigenstates of the quantum Hamiltonians correspond to intersection points of two Lagrangian submanifolds in the $2 N$-dimensional phase space of the classical $N$-body system. One of them is the $N$ dimensional hyperplane with fixed $q_{i}$ 's and the other one is an $N$-dimensional Lagrangian manifold obtained by fixing levels of $N$ classical Hamiltonians. Since their dimensions are complimentary, the intersection set is a finite number of points. It appears that they contain a specific information about eigenstates of the quantum spin chain.

It is natural to conjecture that it is the Yang-Yang (YY) function (yielding solutions of the Bethe equations as its critical points) that characterizes the structure of the intersection set. It was argued in [49] that the YY function plays the role of the generating function for the Lagrangian submanifold in the classical model. However, the meaning of this statement is still to be clarified on particular examples.

The QC duality is traced back to paper [36], where joint spectra of some finitedimensional operators were linked to the classical Toda chain. Later it was extended to the following cases:

a) The Gaudin model (from the quantum side) and the CM many-body system (from the classical side) $[37,38,41]$;

b) Inhomogeneous spin chains of the $X X X$ - and $X X Z$-type with twisted boundary conditions (from the quantum side) and rational or trigonometric RS many-body systems (from the classical side) [39, 40] 
The particles coordinates in the CM or RS models were identified with the inhomogeneities at the sites in the spin chains while eigenvalues of the Lax matrix for the CM or RS models were shown to coincide with eigenvalues of the twist matrix at the spin chain side, with certain multiplicities. The QC duality has been recently discussed in the brane framework in [35] and was related to the duality between quiver $3 \mathrm{~d}$ theory and $4 \mathrm{~d}$ theory at the interval with nontrivial boundary conditions. Moreover, it was suggested that the generalized duality holds when the number of inhomogeneities in the spin chain does not coincide with the number of particles on the classical side. However, the arguments in favour of the QC duality used in all these works were rather indirect.

The main goal of this paper is to give a precise formulation of the $\mathrm{QC}$ duality for a rather representative class of models together with direct proofs. The latter require an elaborate algebraic analysis. We also present the brane counterparts of all its aspects using the brane interpretation of the duality as the relation between the $3 \mathrm{~d}$ quiver gauge SUSY theory and 4d SUSY gauge theory with nontrivial boundary conditions [35].

To be precise, we give a direct proof of the following correspondence between quantum and classical integrable systems (Theorem 1 in section 4 ).

On the quantum side, consider the inhomogeneous $G L(n)$-based generalized spin chain of $X X X$ type with a formal Planck's constant $\hbar$ on $N$ sites with inhomogeneity parameters $q_{i}$ and vector representations at each site. Let us impose twisted boundary conditions with the twist matrix $V=\operatorname{diag}\left(V_{1}, V_{2}, \ldots, V_{n}\right)$, with the generating function of commuting integrals of motion (the transfer matrix) depending on the spectral parameter $z$ being of the form

$$
T^{\mathrm{xxx}}(z)=\operatorname{tr} V+\sum_{j=1}^{N} \frac{H_{j}^{\mathrm{xxx}}}{z-q_{j}} .
$$

The residues $H_{j}^{X X X}$ are (non-local) Hamiltonians of the spin chain. Their eigenvalues depend on the set $\left\{q_{i}\right\}_{N}$ and on a solution $\left\{\left\{\mu_{i}^{1}\right\}_{N_{1}}, \ldots,\left\{\mu_{i}^{n-1}\right\}_{N_{n-1}}\right\}$ of the system of (nested) Bethe equations (BE): $H_{j}^{X X X}=H_{j}^{X X X}\left(\left\{q_{i}\right\}_{N} ;\left\{\mu_{i}^{1}\right\}_{N_{1}}, \ldots,\left\{\mu_{i}^{n-1}\right\}_{N_{n-1}}\right)$, where $N_{a}$ denotes the number of Bethe roots at the a-th level of the nested Bethe ansatz.

On the classical side, consider the RS model with coupling constant $\hbar$ and the number of particles, $N$, equal to the number of sites of the $G L(n)$ spin chain. The Lax matrix of the model is

$$
L_{i j}^{\mathrm{RS}}\left(\left\{\dot{q}_{i}\right\}_{N},\left\{q_{i}\right\}_{N}, \hbar\right)=\frac{\hbar \dot{q}_{j}}{q_{i}-q_{j}+\hbar}, \quad i, j=1, \ldots, N
$$

where $\left\{q_{i}\right\}_{N}$ are coordinates of the particles and $\left\{\dot{q}_{i}\right\}_{N}$ are their velocities.

The claim is that under the substitution

$$
\dot{q}_{j}=\frac{1}{\hbar} H_{j}^{\mathrm{xxx}}\left(\left\{q_{i}\right\}_{N} ;\left\{\mu_{i}^{1}\right\}_{N_{1}}, \ldots,\left\{\mu_{i}^{n-1}\right\}_{N_{n-1}}\right), \quad j=1, \ldots, N,
$$

where the set of $\mu_{i}^{a}$ 's is any solution of the nested BE for the spin chain, the eigenvalues of the Lax matrix are

$$
(\underbrace{V_{1}, \ldots, V_{1}}_{N-N_{1}}, \underbrace{V_{2}, \ldots, V_{2}}_{N_{1}-N_{2}}, \ldots, \underbrace{V_{n-1}, \ldots, V_{n-1}}_{N_{n-2}-N_{n-1}}, \underbrace{V_{n}, \ldots, V_{n}}_{N_{n-1}})
$$


This means that the spectral problem for the quantum spin chain is equivalent to an "inverse spectral problem" for the Lax matrix of the classical RS system: for the matrix of the form (1.1) find velocities $\dot{q}_{i}$ in such a way that the spectrum has the form (1.3).

The simplest example is given in section 4 .

The paper is organized as follows. In section 2 the main properties of the classical many-body systems are summarized. In section 3 we review the relevant facts concerning the quantum spin chains and in section 4 the algebraic analysis yielding the precise correspondence between the data at the classical and quantum sides is presented. The brane picture behind the correspondence considered can be found in section 5. A partial list of open problems is given in the last section.

\section{Acknowledgments}

The authors are grateful to A. Alexandrov, K. Bulycheva, E. Gorsky, S. Gukov, V. Kazakov, P. Koroteev, I. Krichever, S. Leurent, A. Morozov, N. Nekrasov, N. Slavnov, T. Takebe and Z. Tsuboi for discussions. The work of A.G. was supported in part by grants RFBR-1202-00284 and PICS-12-02-91052. A.G. thanks the organizers of Simons Summer School at Simons Center for Geometry and Physics where the part of this work has been done for the hospitality and support. The work of A.Zabrodin was supported in part by RFBR grant 11-02-01220, by joint RFBR grants 12-02-91052-CNRS, 12-02-92108-JSPS and by Ministry of Science and Education of Russian Federation under contract 8207 and by grant NSh3349.2012.2 for support of leading scientific schools. The work of A.Zotov was supported in part by RFBR grants 14-01-00860, by grant NSh-4724.2014.2 for support of leading scientific schools and by the D. Zimin's fund "Dynasty".

\section{Classical integrable many-body systems}

The Ruijsenaars-Schneider (RS) model $[17,18]$ : of $\mathfrak{g l}_{N}$ type is defined by the following $N \times N$ Lax matrix

$$
L_{i j}^{\mathrm{RS}}=\frac{\eta \nu e^{\eta p_{j}}}{q_{i}-q_{j}+\eta \nu} \prod_{k \neq j}^{N} \frac{q_{j}-q_{k}+\eta \nu}{q_{j}-q_{k}}, \quad i, j=1, \ldots, N,
$$

where $p_{i}$ and $q_{i}$ are the canonical variables with the Poisson brackets $\left\{p_{i}, q_{j}\right\}=\delta_{i j}, \nu$ is the coupling constant and $\eta$ is the inverse of the light speed. Note that there is a freedom in definition (2.1) coming from the canonical transformation

$$
e^{\eta p_{j}} \longrightarrow e^{\eta p_{j}} \prod_{k \neq j}\left(\frac{q_{j}-q_{k}+\xi}{q_{j}-q_{k}-\xi}\right)^{g},
$$

where $g$ and $\xi$ are arbitrary constants. The conventional form of the RS Lax matrix $[17,18]$ is reproduced by choosing $\xi= \pm \eta \nu, g=\mp \frac{1}{2}$.

The Hamiltonian of the model is

$$
H^{\mathrm{RS}}=\operatorname{tr} L^{\mathrm{RS}}=\sum_{j=1}^{N} e^{\eta p_{j}} \prod_{k \neq j}^{N} \frac{q_{j}-q_{k}+\eta \nu}{q_{j}-q_{k}} .
$$


The higher Hamiltonians in involution are $H_{k}^{\mathrm{RS}}=\frac{1}{k} \operatorname{tr}\left(L^{\mathrm{RS}}\right)^{k}, H_{1}^{\mathrm{RS}}=H^{\mathrm{RS}}$.

As is seen from (2.3), the velocities are given by

$$
\dot{q}_{j}={\frac{\partial H^{\mathrm{RS}}}{\partial p_{i}}}^{\mathrm{R}}=\eta e^{\eta p_{j}} \prod_{k \neq j}^{N} \frac{q_{j}-q_{k}+\eta \nu}{q_{j}-q_{k}} .
$$

In terms of velocities, the Lax matrix (2.1) takes the form

$$
L_{i j}^{\mathrm{RS}}=\frac{\nu \dot{q}_{j}}{q_{i}-q_{j}+\eta \nu}, \quad i, j=1, \ldots, N .
$$

The equations of motion are:

$$
\ddot{q}_{i}=-\sum_{k \neq i} \frac{2 \eta^{2} \nu^{2} \dot{q}_{i} \dot{q}_{k}}{\left(q_{i}-q_{k}\right)\left(\left(q_{i}-q_{k}\right)^{2}-\eta^{2} \nu^{2}\right)}, \quad i=1, \ldots, N .
$$

In what follows we put $\eta=1$ since it can be easily restored. But before that let us consider the non-relativistic limit $\eta \rightarrow 0$.

The Calogero-Moser (CM) model [13-16]: is defined by the Lax matrix

$$
L_{i j}^{\mathrm{CM}}=\lim _{\eta \rightarrow 0} \frac{L_{i j}^{\mathrm{RS}}-\delta_{i j}}{\eta}=\delta_{i j}\left(p_{i}+\nu \sum_{k \neq i} \frac{1}{q_{i}-q_{k}}\right)+\nu \frac{1-\delta_{i j}}{q_{i}-q_{j}}, \quad i, j=1, \ldots, N .
$$

The $\eta$-expansion of the Hamiltonian $H^{\mathrm{RS}}$ is $H^{\mathrm{RS}}=1+\eta P^{\mathrm{CM}}+\eta^{2} H^{\mathrm{CM}}+O\left(\eta^{3}\right)$, where

$$
\begin{aligned}
P^{\mathrm{CM}} & =\sum_{j=1}^{N}\left(p_{j}+\sum_{k \neq j} \frac{\nu}{q_{j}-q_{k}}\right)=\sum_{j=1}^{N} p_{j}, \\
H^{\mathrm{CM}} & =\frac{1}{2} \operatorname{tr}\left(L^{\mathrm{CM}}\right)^{2}=\frac{1}{2} \sum_{i=1}^{N}\left(p_{i}+\sum_{k \neq i} \frac{\nu}{q_{i}-q_{k}}\right)^{2}-\sum_{i<j}^{N} \frac{\nu^{2}}{\left(q_{i}-q_{j}\right)^{2}}
\end{aligned}
$$

are respectively the total momentum and the Hamiltonian of the CM particles. Similarly to the $\eta \neq 0$ case, there is a freedom to make a canonical transformation of the form

$$
p_{j} \rightarrow p_{j}+\nu^{\prime} \sum_{k \neq j} \frac{1}{q_{j}-q_{k}}
$$

in (2.7), where $\nu^{\prime}$ is an arbitrary constant. The conventional form of the CM Lax matrix corresponds to the choice $\nu^{\prime}=-\nu$. The higher Hamiltonians in involution are $H_{k}^{\mathrm{CM}}=$ $\frac{1}{k} \operatorname{tr}\left(L^{\mathrm{CM}}\right)^{k}$, with $H_{1}^{\mathrm{CM}}=P^{\mathrm{CM}}, H_{2}^{\mathrm{CM}}=H^{\mathrm{CM}}$.

The particles velocities are

$$
\dot{q}_{i}=\frac{\partial H^{\mathrm{CM}}}{\partial p_{i}}=p_{i}+\sum_{k \neq i} \frac{\nu}{q_{i}-q_{k}} .
$$

In terms of the velocities, the Lax matrix and the equations of motion acquire their conventional form:

$$
\begin{aligned}
L_{i j}^{\mathrm{CM}} & =\delta_{i j} \dot{q}_{i}+\nu \frac{1-\delta_{i j}}{q_{i}-q_{j}}, \quad i, j=1, \ldots, N, \\
\ddot{q}_{i} & =-\sum_{k \neq i} \frac{2 \nu^{2}}{\left(q_{i}-q_{k}\right)^{3}}, \quad i=1, \ldots, N .
\end{aligned}
$$




\section{Quantum spin chains and Gaudin models}

The generalized $G L(n)$-invariant inhomogeneous $X X X$ spin chain [50]. The Hilbert space $\mathcal{H}$ of the model is the tensor product of the highest weight $\mathfrak{g l}_{n}$-modules $\mathcal{M}_{1} \otimes \ldots \otimes \mathcal{M}_{N}$ with the highest weights $\lambda^{(1)}, \ldots, \lambda^{(N)}, \lambda^{(i)}=\left(\lambda_{1}^{(i)}, \ldots, \lambda_{n}^{(i)}\right)$ with $\lambda_{1}^{(i)} \geq$ $\lambda_{2}^{(i)} \geq \ldots \geq \lambda_{n}^{(i)} \geq 0$. Let $\Lambda=\left\{\lambda^{(1)}, \ldots, \lambda^{(N)}\right\}$ be the set of the highest weights. By $\mathcal{M}_{0}=\mathbb{C}^{n}$ we denote the auxiliary space of the vector $G L(n)$-representation with the highest weight $\lambda^{(0)}=(1,0, \ldots, 0)$.

The $G L(n)$-invariant $R$-matrix $R_{0 j}(z)$ acts non-trivially in $\mathcal{M}_{0} \otimes \mathcal{M}_{j}$. It has the form

$$
R_{0 j}(z)=1 \otimes 1+\frac{\hbar}{z} \sum_{a, b=1}^{n} E_{a b}^{(0)} \otimes \mathrm{E}_{b a}^{(j)},
$$

where $\left(E_{a b}^{(0)}\right)_{c d}=\delta_{a c} \delta_{b d}$ are basic matrices in the auxiliary space and $\mathrm{E}_{a b}^{(j)}$ are generators of $\mathfrak{g l}_{n}$ acting in $\mathcal{M}_{j}$ with the standard commutation relations $\left[\mathrm{E}_{a b}^{(j)}, \mathrm{E}_{a^{\prime} b^{\prime}}^{(j)}\right]=\delta_{a^{\prime} b} \mathrm{E}_{a b^{\prime}}^{(j)}-\delta_{a b^{\prime}} \mathrm{E}_{a^{\prime} b}^{(j)}$.

The quantum transfer matrix is an operator in $\mathcal{H}$ defined as trace of a product of the $R$ matrices and the twist matrix $V=\operatorname{diag}\left(V_{1}, \ldots, V_{n}\right) \in G L(n)$ taken in the auxiliary space:

$$
\hat{T}_{\Lambda}^{\mathrm{xxx}}(z)=\operatorname{tr}_{0}\left[V_{0} R_{01}\left(z-q_{1}\right) \ldots R_{0 N}\left(z-q_{N}\right)\right],
$$

where we write $V_{0}$ instead of $V$ to stress that this matrix acts in $\mathcal{M}_{0}$. Sometimes we will use the more detailed notation $\hat{T}_{\Lambda}^{\mathrm{xxx}}(z)=\hat{T}_{\Lambda}^{\mathrm{xxx}}\left(z ;\left\{q_{i}\right\}, V, \hbar\right)$. Hereafter we assume that the inhomogeneity parameters $q_{i}$ are all distinct. The Yang-Baxter equation satisfied by the $R$-matrix and the $G L(n)$-invariance of the $R$-matrix imply that the transfer matrices $\hat{T}_{\Lambda}^{\mathrm{xxx}}(z)$ with the same $\left\{q_{i}\right\}, \Lambda, \hbar$ and $V$ commute for all values of $z$. Therefore, the transfer matrix can serve as a generating function for commuting quantum Hamiltonians. It is clear from (3.2) that $\hat{T}_{\Lambda}^{\mathrm{xxx}}(z)$ has simple poles at $z=q_{i}$. The Hamiltonians can be defined as residues at these poles:

$$
\hat{H}_{\Lambda, i}^{\mathrm{xxx}}:=\operatorname{Res}_{z=q_{i}} \hat{T}_{\Lambda}^{\mathrm{xxx}}(z) .
$$

In general, they are non-local operators involving spins at all sites of the chain.

As is shown in [50] (see also [51-53]), the eigenvalues of the transfer matrix and the Hamiltonians are of the form

$$
\begin{aligned}
T_{\Lambda}^{\mathrm{xxx}}(z) & =\sum_{b=1}^{n} V_{b} \prod_{k=1}^{N} \frac{z-q_{k}+\hbar \lambda_{b}^{(k)}}{z-q_{k}} \prod_{\gamma=1}^{N_{b-1}} \frac{z-\mu_{\gamma}^{b-1}+\hbar}{z-\mu_{\gamma}^{b-1}} \prod_{\gamma=1}^{N_{b}} \frac{z-\mu_{\gamma}^{b}-\hbar}{z-\mu_{\gamma}^{b}} \\
\frac{1}{\hbar} H_{\Lambda, i}^{\mathrm{xxx}} & =\sum_{b=1}^{n} V_{b} \lambda_{b}^{(i)} \prod_{k \neq i}^{N} \frac{q_{i}-q_{k}+\hbar \lambda_{b}^{(k)}}{q_{i}-q_{k}} \prod_{\gamma=1}^{N_{b-1}} \frac{q_{i}-\mu_{\gamma}^{b-1}+\hbar}{q_{i}-\mu_{\gamma}^{b-1}} \prod_{\gamma=1}^{N_{b}} \frac{q_{i}-\mu_{\gamma}^{b}-\hbar}{q_{i}-\mu_{\gamma}^{b}}
\end{aligned}
$$

where the parameters $\mu_{\gamma}^{b}$ satisfy the system of the nested BE:

$$
V_{b} \prod_{k=1}^{N} \frac{\mu_{\beta}^{b}-q_{k}+\hbar \lambda_{b}^{(k)}}{\mu_{\beta}^{b}-q_{k}+\hbar \lambda_{b+1}^{(k)}} \prod_{\gamma=1}^{N_{b-1}} \frac{\mu_{\beta}^{b}-\mu_{\gamma}^{b-1}+\hbar}{\mu_{\beta}^{b}-\mu_{\gamma}^{b-1}}=V_{b+1} \prod_{\gamma \neq \beta}^{N_{b}} \frac{\mu_{\beta}^{b}-\mu_{\gamma}^{b}+\hbar}{\mu_{\beta}^{b}-\mu_{\gamma}^{b}-\hbar} \prod_{\gamma=1}^{N_{b+1}} \frac{\mu_{\beta}^{b}-\mu_{\gamma}^{b+1}-\hbar}{\mu_{\beta}^{b}-\mu_{\gamma}^{b+1}} .
$$


Here $b=1, \ldots, n-1, \beta=1, \ldots, N_{b}$. It is convenient to put $N_{0}=N_{n}=0$. The total number of equations equals $\sum_{b=1}^{n-1} N_{b}$. We also have [50,52, 53]: $N \geq N_{1} \geq N_{2} \geq \ldots \geq N_{n-1} \geq 0$.

It is known $[52,53]$ that the operators

$$
\hat{M}_{a}=\sum_{j=1}^{N} \mathrm{E}_{a a}^{(j)}, \quad a=1, \ldots, n,
$$

commute with the transfer matrix. The eigenvectors of the latter, built from solutions to the BE with the numbers of Bethe roots at level $b$ equal to $N_{b}$, are also eigenvectors of the operators $\hat{M}_{a}$ with the eigenvalues

$$
M_{a}=N_{a-1}-N_{a}+\sum_{j=1}^{N} \lambda_{a}^{(j)}
$$

In what follows we consider the important particular case of vector representations of $G L(n)$ at all sites of the chain, i.e.,

$$
\lambda^{(i)}=(1,0, \ldots, 0), \text { for all } i=1, \ldots, N .
$$

In this case we will simply write $\hat{T}^{\mathrm{xxx}}(z)$ and $\hat{H}_{i}^{\mathrm{xxx}}$ for the transfer matrix and Hamiltonians. Then all terms in (3.5) vanish except the first one:

$$
\frac{1}{\hbar} H_{i}^{\mathrm{xxx}}=V_{1} \prod_{k=1}^{N} \frac{q_{i}-q_{k}+\hbar}{q_{i}-q_{k}} \prod_{\gamma=1}^{N_{1}} \frac{q_{i}-\mu_{\gamma}^{1}-\hbar}{q_{i}-\mu_{\gamma}^{1}} .
$$

The eigenvalues of the operators $\hat{M}_{a}$ are: $M_{1}=N-N_{1}, M_{a}=N_{a-1}-N_{a}, a=2, \ldots, n$. The BE simplify as well because the first product in the l.h.s. of (3.6) is non-trivial only at $b=1$. The BE (3.6) are naturally divided into $n-1$ groups:

$$
\begin{aligned}
& B E_{1}: \quad V_{1} \prod_{k=1}^{N} \frac{\mu_{\beta}^{1}-q_{k}+\hbar}{\mu_{\beta}^{1}-q_{k}}=V_{2} \prod_{\gamma \neq \beta}^{N_{1}} \frac{\mu_{\beta}^{1}-\mu_{\gamma}^{1}+\hbar}{\mu_{\beta}^{1}-\mu_{\gamma}^{1}-\hbar} \prod_{\gamma=1}^{N_{2}} \frac{\mu_{\beta}^{1}-\mu_{\gamma}^{2}-\hbar}{\mu_{\beta}^{1}-\mu_{\gamma}^{2}}, \\
& B E_{b}: \quad V_{b} \prod_{\gamma=1}^{N_{b-1}} \frac{\mu_{\beta}^{b}-\mu_{\gamma}^{b-1}+\hbar}{\mu_{\beta}^{b}-\mu_{\gamma}^{b-1}}=V_{b+1} \prod_{\gamma \neq \beta}^{N_{b}} \frac{\mu_{\beta}^{b}-\mu_{\gamma}^{b}+\hbar}{\mu_{\beta}^{b}-\mu_{\gamma}^{b}-\hbar} \prod_{\gamma=1}^{N_{b+1}} \frac{\mu_{\beta}^{b}-\mu_{\gamma}^{b+1}-\hbar}{\mu_{\beta}^{b}-\mu_{\gamma}^{b+1}}
\end{aligned}
$$

for $b=2, \ldots, n-2$ and

$$
B E_{n-1}: \quad V_{n-1} \prod_{\gamma=1}^{N_{n-2}} \frac{\mu_{\beta}^{n-1}-\mu_{\gamma}^{n-2}+\hbar}{\mu_{\beta}^{n-1}-\mu_{\gamma}^{n-2}}=V_{n} \prod_{\gamma \neq \beta}^{N_{n-1}} \frac{\mu_{\beta}^{n-1}-\mu_{\gamma}^{n-1}+\hbar}{\mu_{\beta}^{n-1}-\mu_{\gamma}^{n-1}-\hbar} .
$$

In what follows we will use the notation $H_{i}^{\mathrm{xxx}}\left(\left\{q_{i}\right\}_{N},\left\{\mu_{\alpha}^{1}\right\}_{N_{1}}\right)$ for the function given by the r.h.s. of (3.10). When the set $\left\{\mu_{\alpha}^{1}\right\}_{N_{1}}$ is taken from a solution to the system of BE, this function is equal to an eigenvalue of the Hamiltonian. 
Example: $\boldsymbol{G L}(\mathbf{2}) \mathbf{X X X}$ chain. In this case the twist matrix is $V=\left(\begin{array}{cc}e^{\omega} & 0 \\ 0 & e^{-\omega}\end{array}\right)$ and eigenvalues of the transfer matrix and the Hamiltonians are given by

$$
\begin{aligned}
& T_{\Lambda}^{\mathrm{xxx}}(z)=e^{\omega} \prod_{k=1}^{N} \frac{z-q_{k}+\hbar \lambda_{1}^{(k)}}{z-q_{k}} \prod_{\gamma=1}^{N_{1}} \frac{z-\mu_{\gamma}-\hbar}{z-\mu_{\gamma}} \\
&+e^{-\omega} \prod_{k=1}^{N} \frac{z-q_{k}+\hbar \lambda_{2}^{(k)}}{z-q_{k}} \prod_{\gamma=1}^{N_{1}} \frac{z-\mu_{\gamma}+\hbar}{z-\mu_{\gamma}} \\
& \frac{1}{\hbar} H_{\Lambda, i}^{\mathrm{xxx}}=e^{\omega} \lambda_{1}^{(i)} \prod_{k \neq i}^{N} \frac{q_{i}-q_{k}+\hbar \lambda_{1}^{(k)}}{q_{i}-q_{k}} \prod_{\gamma=1}^{N_{1}} \frac{q_{i}-\mu_{\gamma}-\hbar}{q_{i}-\mu_{\gamma}} \\
&+e^{-\omega} \lambda_{2}^{(i)} \prod_{k \neq i}^{N} \frac{q_{i}-q_{k}+\hbar \lambda_{2}^{(k)}}{q_{i}-q_{k}} \prod_{\gamma=1}^{N_{1}} \frac{q_{i}-\mu_{\gamma}+\hbar}{q_{i}-\mu_{\gamma}}
\end{aligned}
$$

with the $\mathrm{BE}$ of the form

$$
e^{2 \omega} \prod_{k=1}^{N} \frac{\mu_{\alpha}-q_{k}+\hbar \lambda_{1}^{(k)}}{\mu_{\alpha}-q_{k}+\hbar \lambda_{2}^{(k)}}=\prod_{\gamma \neq \alpha}^{N_{1}} \frac{\mu_{\alpha}-\mu_{\gamma}+\hbar}{\mu_{\alpha}-\mu_{\gamma}-\hbar}, \quad \alpha=1, \ldots, N_{1} .
$$

With the choice $\left(\lambda_{1}^{(i)}, \lambda_{2}^{(i)}\right)=(1,0)$ for all $i=1, \ldots, N$ ( $\operatorname{spin} \frac{1}{2}$ at each site) the second term in (3.15) vanishes and we get

$$
\frac{1}{\hbar} H_{i}^{\mathrm{xxx}}=e^{\omega} \prod_{k \neq i}^{N} \frac{q_{i}-q_{k}+\hbar}{q_{i}-q_{k}} \prod_{\gamma=1}^{N_{1}} \frac{q_{i}-\mu_{\gamma}-\hbar}{q_{i}-\mu_{\gamma}}, \quad i=1, \ldots, N,
$$

where $\mu_{\alpha}$ 's satisfy the BE

$$
e^{2 \omega} \prod_{k=1}^{N} \frac{\mu_{\alpha}-q_{k}+\hbar}{\mu_{\alpha}-q_{k}}=\prod_{\gamma \neq \alpha}^{N_{1}} \frac{\mu_{\alpha}-\mu_{\gamma}+\hbar}{\mu_{\alpha}-\mu_{\gamma}-\hbar}, \quad \alpha=1, \ldots, N_{1} .
$$

The rational $\mathfrak{g l}_{n}$ Gaudin model $[11, \mathbf{1 2}]:$ is the $\varepsilon \rightarrow 0$ limit of the inhomogeneous $X X X$ spin chain with the transfer matrix $\hat{T}_{\Lambda}^{\mathrm{xxx}}\left(z ;\left\{q_{i}\right\}, V^{\varepsilon}, \varepsilon \hbar\right)$. The expansion as $\varepsilon \rightarrow 0$ is

$$
\hat{T}_{\Lambda}^{\mathrm{xxx}}\left(z ;\left\{q_{i}\right\}, V^{\varepsilon}, \varepsilon \hbar\right)=n+\varepsilon\left(\operatorname{tr} v+\sum_{i=1}^{N} \frac{\hbar C_{1}^{(i)}}{z-q_{i}}\right)+\varepsilon^{2}\left(\frac{1}{2} \operatorname{tr} v^{2}+\sum_{i} \frac{\hbar \hat{H}_{\Lambda, i}^{\mathrm{G}}}{z-q_{i}}\right)+O\left(\varepsilon^{3}\right)
$$

where $v=\log V, C_{1}^{(i)}=\sum_{a} \mathrm{E}_{a a}^{(i)}=\sum_{a} \lambda_{a}^{(i)}$ is the first Casimir operator of $\mathrm{U}\left(\mathfrak{g l}_{n}\right)$ at the $i$-th site and

$$
\hat{H}_{\Lambda, i}^{\mathrm{G}}=\sum_{a} v_{a} \mathrm{E}_{a a}^{(i)}+\sum_{j \neq i} \frac{\hbar}{q_{i}-q_{j}} \sum_{a b} \mathrm{E}_{a b}^{(i)} \mathrm{E}_{b a}^{(j)}=\lim _{\varepsilon \rightarrow 0} \frac{\hat{H}_{\Lambda, i}^{\mathrm{xxx}}\left(\left\{q_{i}\right\}, e^{\varepsilon v}, \varepsilon \hbar\right)-\varepsilon \hbar C_{1}^{(i)}}{\hbar \varepsilon^{2}} .
$$

These operators are called Gaudin Hamiltonians. Their eigenvalues can be found by substituting (3.5) into (3.20) and tending $\varepsilon \rightarrow 0$. This gives

$$
H_{\Lambda, i}^{\mathrm{G}}=\sum_{b=1}^{n}\left(v_{b}+\sum_{k \neq i}^{N} \frac{\hbar \lambda_{b}^{(k)} \lambda_{b}^{(i)}}{q_{i}-q_{k}}+\sum_{\gamma=1}^{N_{b-1}} \frac{\hbar \lambda_{b}^{(i)}}{q_{i}-\mu_{\gamma}^{b-1}}-\sum_{\gamma=1}^{N_{b}} \frac{\hbar \lambda_{b}^{(i)}}{q_{i}-\mu_{\gamma}^{b}}\right)
$$


with the BE of the form

$$
v_{b}-v_{b+1}+\sum_{k=1}^{N} \frac{\hbar\left(\lambda_{b}^{(k)}-\lambda_{b+1}^{(k)}\right)}{\mu_{\beta}^{b}-q_{k}}=-\sum_{\gamma=1}^{N_{b-1}} \frac{\hbar}{\mu_{\beta}^{b}-\mu_{\gamma}^{b-1}}+2 \sum_{\gamma \neq \beta}^{N_{b}} \frac{\hbar}{\mu_{\beta}^{b}-\mu_{\gamma}^{b}}-\sum_{\gamma=1}^{N_{b+1}} \frac{\hbar}{\mu_{\beta}^{b}-\mu_{\gamma}^{b+1}},
$$

where $b=1, \ldots, n-1, \beta=1, \ldots, N_{b} N_{0}=N_{n}=0$. By analogy with the $X X X$ spin chain we call the matrix $v=\operatorname{diag}\left(v_{1}, \ldots, v_{n}\right)$ the twist matrix of the Gaudin model. In the context of the Gaudin model, the parameters $q_{i}$ are often called marked points.

The operators $\hat{M}_{a}$ and their eigenvalues on the eigenstates of the Gaudin Hamiltonians are are given by the same formulas $(3.7),(3.8)$. In the case $\lambda^{(i)}=(1,0, \ldots, 0)$ for all $i$ the above formulas simplify:

$$
H_{i}^{\mathrm{G}}=v_{1}+\sum_{k \neq i}^{N} \frac{\hbar}{q_{i}-q_{k}}-\sum_{\gamma=1}^{N_{1}} \frac{\hbar}{q_{i}-\mu_{\gamma}^{1}}
$$

with the $B E_{b}$ :

$$
v_{b}-v_{b+1}+\delta_{1 b} \sum_{k=1}^{N} \frac{\hbar}{\mu_{\beta}^{b}-q_{k}}=-\sum_{\gamma=1}^{N_{b-1}} \frac{\hbar}{\mu_{\beta}^{b}-\mu_{\gamma}^{b-1}}+2 \sum_{\gamma \neq \beta}^{N_{b}} \frac{\hbar}{\mu_{\beta}^{b}-\mu_{\gamma}^{b}}-\sum_{\gamma=1}^{N_{b+1}} \frac{\hbar}{\mu_{\beta}^{b}-\mu_{\gamma}^{b+1}}
$$

The eigenvalues of the operators $\hat{M}_{a}$ are: $M_{1}=N-N_{1}, M_{a}=N_{a-1}-N_{a}, a=2, \ldots, n$. Similarly to the $X X X$ spin chain case, we will use the notation $H_{i}^{\mathrm{G}}\left(\left\{q_{i}\right\}_{N},\left\{\mu_{\alpha}^{1}\right\}_{N_{1}}\right)$ for the function given by the r.h.s. of (3.23). When the set $\left\{\mu_{\alpha}^{1}\right\}_{N_{1}}$ is taken from a solution to the system of $\mathrm{BE}$ (3.22), this function is equal to an eigenvalue of the Hamiltonian.

Example: the rational $\mathfrak{g l}_{2}$ Gaudin model. For the $\mathfrak{g l}_{2}$ Gaudin model with the twist matrix $v=\left(\begin{array}{cc}\omega & 0 \\ 0 & -\omega\end{array}\right)$ equations (3.23) and (3.24) read

$$
\begin{aligned}
\frac{1}{\hbar} H_{i}^{\mathrm{G}} & =\omega+\sum_{k \neq i}^{N} \frac{\hbar}{q_{i}-q_{k}}+\sum_{\gamma=1}^{N_{1}} \frac{\hbar}{\mu_{\gamma}-q_{i}}, \\
2 \omega+\hbar \sum_{k=1}^{N} \frac{1}{\mu_{\alpha}-q_{k}} & =2 \hbar \sum_{\gamma \neq \alpha}^{N_{1}} \frac{1}{\mu_{\alpha}-\mu_{\gamma}}
\end{aligned}
$$

for all $\alpha=1, \ldots, M$. Here $\mu_{\alpha}=\mu_{\alpha}^{1}$.

\section{The QC duality}

In this section we derive the relation between the spectrum of the quantum $X X X$ spin chain Hamiltonians and the spectrum of the classical RS Lax matrix which is the basis of the QC duality. Our main statement is the following theorem.

Theorem 1. Given the Lax matrix (2.5) of the $\mathfrak{g l}_{N} R S$ model

$$
L_{i j}^{R S}\left(\{\dot{q}\}_{N},\{q\}_{N}, \nu\right)=\frac{\nu \dot{q}_{j}}{q_{i}-q_{j}+\nu}, \quad i, j=1, \ldots, N
$$


make the substitution

$$
\nu=\hbar \quad \text { and } \quad \dot{q}_{j}=\frac{1}{\hbar} H_{j}^{X X X}\left(\left\{q_{i}\right\}_{N},\left\{\mu_{\alpha}^{1}\right\}_{N_{1}}\right)
$$

where the r.h.s. is given by (3.10). If the $N_{1}$ parameters $\mu_{\alpha}^{1}$ are taken from any solution $\left\{\left\{\mu_{\alpha}^{1}\right\}_{N_{1}}, \ldots\left\{\mu_{\alpha}^{n-1}\right\}_{N_{n-1}}\right\}$ to the system of BE (3.11)-(3.13) for the inhomogeneous spin chain on $N \geq n$ sites with inhomogeneity parameters $q_{i}$ and the twist matrix $V=\operatorname{diag}\left(V_{1}, \ldots, V_{n}\right)$, then the spectrum of the Lax matrix has the following form:

$$
\begin{aligned}
\left.\operatorname{Spec} L^{R S}\left(\frac{1}{\hbar}\left\{H_{j}^{X X X}\right\}_{N},\left\{q_{j}\right\}_{N}, \hbar\right)\right|_{\mathrm{BE}} \\
=(\underbrace{V_{1}, \ldots, V_{1}}_{N-N_{1}}, \underbrace{V_{2}, \ldots, V_{2}}_{N_{1}-N_{2}}, \ldots, \underbrace{V_{n-1}, \ldots, V_{n-1}}_{N_{n-2}-N_{n-1}}, \underbrace{V_{n}, \ldots, V_{n}}_{N_{n-1}})
\end{aligned}
$$

The rest of this section is devoted to the proof of the theorem. But before passing to the proof, let us say a few words about the meaning of this statement. It implies, in particular, that one can solve the spectral problem for the Hamiltonians of the inhomogeneous spin chain without addressing the BE at any step but by solving an "inverse spectral problem" for the Lax matrix $L^{\mathrm{RS}}$ of the classical RS system of particles. More precisely, let $\left\{q_{i}\right\}_{N}$ be the inhomogeneity parameters of the spin chain with the Planck's constant $\hbar$ and $V$ its twist matrix. Let the eigenvalues of the RS Lax matrix be equal to the eigenvalues $V_{a}$ of the twist matrix, with some multiplicities $M_{a} \geq 0$ such that $\sum_{a} M_{a}=N$. (This fixes values of all the RS Hamiltonians: $H_{k}^{\mathrm{RS}}=\frac{1}{k} \sum_{a} M_{a} V_{a}^{k}$.) Then the spectrum of $\hat{H}_{j}^{\mathrm{xxx}}$ in the sector where eigenvalues of the operators $\hat{M}_{a}$ are equal to $M_{a}$ is given by the values of $H_{j}^{\mathrm{xxx}}$ such that the matrix $L_{i j}^{\mathrm{RS}}=\frac{H_{j}^{\mathrm{xxx}}}{q_{i}-q_{j}+\hbar}$ has the prescribed spectrum. Here we assume that each site carries the vector representation of $G L(N)$. We anticipate that this approach can be extended to the spin chains with arbitrary highest weight representations at sites.

The proof will use the results of [54]. In order to prove the statement, i.e.,

$$
\operatorname{det}\left[\left.L^{\mathrm{RS}}\left(\frac{1}{\hbar}\left\{H_{j}^{\mathrm{xxx}}\right\}_{N},\left\{q_{j}\right\}_{N}, \hbar\right)\right|_{\mathrm{BE}}-\lambda\right]=\prod_{a=1}^{n}\left(V_{a}-\lambda\right)^{M_{a}},
$$

where $M_{1}=N-N_{1}, M_{a}=N_{a-1}-N_{a}(2 \leq a \leq n)$ let us introduce the following pair of matrices:

$$
\mathcal{L}_{i j}\left(\left\{x_{i}\right\}_{N},\left\{y_{i}\right\}_{M}, g\right)=\frac{g \hbar}{x_{i}-x_{j}+\hbar} \prod_{k \neq j}^{N} \frac{x_{j}-x_{k}+\hbar}{x_{j}-x_{k}} \prod_{\gamma=1}^{M} \frac{x_{j}-y_{\gamma}}{x_{j}-y_{\gamma}+\hbar}, \quad i, j=1, \ldots, N
$$

and

$$
\widetilde{\mathcal{L}}_{\alpha \beta}\left(\left\{y_{i}\right\}_{M},\left\{x_{i}\right\}_{N}, g\right)=\frac{g \hbar}{y_{\alpha}-y_{\beta}+\hbar} \prod_{\gamma \neq \beta}^{M} \frac{y_{\beta}-y_{\gamma}-\hbar}{y_{\beta}-y_{\gamma}} \prod_{k=1}^{N} \frac{y_{\beta}-x_{k}}{y_{\beta}-x_{k}-\hbar}, \quad \alpha, \beta=1, \ldots, M,
$$

From the computational point of view the QC duality is based on the following algebraic relation between $\mathcal{L}$ and $\widetilde{\mathcal{L}}$ : 
Proposition 4.1. For the pair of matrices (4.4) and (4.5) it holds:

$\operatorname{det}_{N \times N}\left(\mathcal{L}\left(\left\{x_{i}\right\}_{N},\left\{y_{i}\right\}_{M}, g\right)-\lambda\right)=(g-\lambda)^{N-M} \operatorname{det}_{M \times M}\left(\widetilde{\mathcal{L}}\left(\left\{y_{i}\right\}_{M},\left\{x_{i}\right\}_{N}, g\right)-\lambda\right)$

The proof of (4.6) is given in the appendix.

Proof of Theorem 1. The proof of (4.3) includes $n-1$ steps and consists in successive application of (4.6) and taking into account the BE (3.11)-(3.13). Indeed, set

$$
\begin{aligned}
L_{i j}^{(0)} & =L_{i j}^{\mathrm{RS}}\left(\frac{1}{\hbar}\left\{H_{j}^{\mathrm{xxx}}\right\}_{N},\left\{q_{i}\right\}_{N}, \hbar\right)=\frac{\hbar V_{1}}{q_{i}-q_{j}+\hbar} \prod_{k \neq j}^{N} \frac{q_{j}-q_{k}+\hbar}{q_{j}-q_{k}} \prod_{\gamma=1}^{N_{1}} \frac{q_{j}-\mu_{\gamma}^{1}-\hbar}{q_{j}-\mu_{\gamma}^{1}} \\
& =\mathcal{L}_{i j}\left(\left\{q_{i}-\hbar\right\}_{N},\left\{\mu_{\alpha}^{1}\right\}_{N_{1}}, V_{1}\right),
\end{aligned}
$$

and define (at the first step)

$$
L_{\alpha \beta}^{(1)}=\widetilde{\mathcal{L}}_{\alpha \beta}\left(\left\{\mu_{\alpha}^{1}\right\}_{N_{1}},\left\{q_{i}-\hbar\right\}_{N}, V_{1}\right)=\frac{\hbar V_{1}}{\mu_{\alpha}^{1}-\mu_{\beta}^{1}+\hbar} \prod_{\gamma \neq \beta}^{N_{1}} \frac{\mu_{\beta}^{1}-\mu_{\gamma}^{1}-\hbar}{\mu_{\beta}^{1}-\mu_{\gamma}^{1}} \prod_{k=1}^{N} \frac{\mu_{\beta}^{1}-q_{k}+\hbar}{\mu_{\beta}^{1}-q_{k}},
$$

where $\alpha, \beta=1, \ldots, N_{1}$. Equation (4.6) implies that

$$
\operatorname{det}_{N \times N}\left(L^{(0)}-\lambda\right)=\left(V_{1}-\lambda\right)^{N-N_{1}} \operatorname{det}_{N_{1} \times N_{1}}\left(L^{(1)}-\lambda\right) .
$$

Next, impose BE (3.11) to get:

$$
\left.L_{\alpha \beta}^{(1)}\right|_{\mathrm{BE}_{1}}=\frac{\hbar V_{2}}{\mu_{\alpha}^{1}-\mu_{\beta}^{1}+\hbar} \prod_{\gamma \neq \beta}^{N_{1}} \frac{\mu_{\beta}^{1}-\mu_{\gamma}^{1}+\hbar}{\mu_{\beta}^{1}-\mu_{\gamma}^{1}} \prod_{\gamma=1}^{N_{2}} \frac{\mu_{\beta}^{1}-\mu_{\gamma}^{2}-\hbar}{\mu_{\beta}^{1}-\mu_{\gamma}^{2}}, \quad \alpha, \beta=1, \ldots, N_{1},
$$

i.e.,

$$
\left.L^{(1)}\right|_{\mathrm{BE}_{1}}=\mathcal{L}\left(\left\{\mu_{\alpha}^{1}-\hbar\right\}_{N_{1}},\left\{\mu_{\alpha}^{2}\right\}_{N_{2}}, V_{2}\right) .
$$

At the second step we define

$$
L_{\alpha \beta}^{(2)}=\widetilde{\mathcal{L}}_{\alpha \beta}\left(\left\{\mu_{\gamma}^{2}\right\}_{N_{2}},\left\{\mu_{\gamma}^{1}-\hbar\right\}_{N_{1}}, V_{2}\right), \quad \alpha, \beta=1, \ldots, N_{2},
$$

and, similarly to the previous step, we use (4.6) and BE (3.12) to get:

$$
\begin{aligned}
\operatorname{det}_{N_{1} \times N_{1}}\left(L^{(1)}-\lambda\right) & =\left(V_{2}-\lambda\right)^{N_{1}-N_{2}} \operatorname{det}_{N_{2} \times N_{2}}\left(L^{(2)}-\lambda\right), \\
\left.L^{(2)}\right|_{\mathrm{BE}_{2}} & =\mathcal{L}\left(\left\{\mu_{\alpha}^{2}-\hbar\right\}_{N_{2}},\left\{\mu_{\alpha}^{3}\right\}_{N_{3}}, V_{3}\right) .
\end{aligned}
$$

and so on until the last step, where we use (3.13):

$$
\left.L_{\alpha \beta}^{(n-1)}\right|_{\mathrm{BE}_{n-1}}=\frac{\hbar V_{n}}{\mu_{\alpha}^{n-1}-\mu_{\beta}^{n-1}+\hbar} \prod_{\gamma \neq \beta}^{N_{n-1}} \frac{\mu_{\beta}^{n-1}-\mu_{\gamma}^{n-1}+\hbar}{\mu_{\beta}^{n-1}-\mu_{\gamma}^{n-1}}, \quad \alpha, \beta=1, \ldots, N_{n-1} .
$$

The latter matrix obeys the equation $\operatorname{det}_{N_{n-1} \times N_{n-1}}\left(L^{(n-1)}-\lambda\right)=\left(V_{n}-\lambda\right)^{N_{n-1}}$ which follows from Proposition 4.1 for $N=N_{n-1}$ and $M=0$. 
In the limiting case we have the $\mathrm{QC}$ duality between the quantum Gaudin and the classical CM models. The following analogue of Theorem 1 holds true.

Theorem 2. Given the Lax matrix (2.12) of the $\mathfrak{g l}_{N} C M$ model

$$
L_{i j}^{C M}=\delta_{i j} \dot{q}_{i}+\nu \frac{1-\delta_{i j}}{q_{i}-q_{j}}, \quad i, j=1, \ldots, N
$$

make the substitution

$$
\nu=\hbar \quad \text { and } \quad \dot{q}_{j}=\frac{1}{\hbar} H_{j}^{G}\left(\left\{q_{i}\right\}_{N},\left\{\mu_{\alpha}^{1}\right\}_{N_{1}}\right), \quad j=1, \ldots, N,
$$

where the r.h.s. is given by (3.23). If the $N_{1}$ parameters $\mu_{\alpha}^{1}$ are taken from any solution $\left\{\left\{\mu_{\alpha}^{1}\right\}_{N_{1}}, \ldots\left\{\mu_{\alpha}^{n-1}\right\}_{N_{n-1}}\right\}$ to the system of BE (3.24) for the $\mathfrak{g l}_{n}$ Gaudin model with $N \geq n$ marked points $q_{i}$ and the twist matrix $v=\operatorname{diag}\left(v_{1}, \ldots, v_{n}\right)$, then the spectrum of the Lax matrix has the following form:

$$
\begin{aligned}
\left.\operatorname{Spec} L^{C M}\left(\frac{1}{\hbar}\left\{H_{j}^{G}\right\}_{N},\left\{q_{j}\right\}_{N}, \hbar\right)\right|_{\mathrm{BE}} & \\
= & (\underbrace{v_{1}, \ldots, v_{1}}_{N-N_{1}}, \underbrace{v_{2}, \ldots, v_{2}}_{N_{1}-N_{2}}, \ldots, \underbrace{v_{n-1}, \ldots, v_{n-1}}_{N_{n-2}-N_{n-1}}, \underbrace{v_{n}, \ldots, v_{n}}_{N_{n-1}})
\end{aligned}
$$

The proof is based on the analogue of Proposition 4.1. Introduce the pair of matrices

$$
\mathcal{L}_{i j}\left(\left\{x_{i}\right\}_{N},\left\{y_{i}\right\}_{M}, \omega\right)=\delta_{i j}\left(\omega+\sum_{k \neq i}^{N} \frac{\hbar}{q_{i}-q_{k}}+\sum_{\gamma=1}^{M} \frac{\hbar}{\mu_{\gamma}-q_{i}}\right)+\left(1-\delta_{i j}\right) \frac{\hbar}{q_{i}-q_{j}},
$$

where $i, j=1, \ldots, N$ and

$$
\widetilde{\mathcal{L}}_{\alpha \beta}\left(\left\{y_{i}\right\}_{M},\left\{x_{i}\right\}_{N}, \omega\right)=\delta_{\alpha \beta}\left(\omega-\sum_{\gamma \neq \alpha}^{M} \frac{\hbar}{\mu_{\alpha}-\mu_{\gamma}}-\sum_{k=1}^{N} \frac{\hbar}{q_{k}-\mu_{\alpha}}\right)+\left(1-\delta_{\alpha \beta}\right) \frac{\hbar}{\mu_{\alpha}-\mu_{\beta}},
$$

where $\alpha, \beta=1, \ldots, M$. The relation between them is given by

Proposition 4.2. For the pair of matrices (4.19) and (4.20) it holds:

$$
\operatorname{det}_{N \times N}\left(\mathcal{L}\left(\left\{x_{i}\right\}_{N},\left\{y_{i}\right\}_{M}, \omega\right)-\lambda\right)=(\omega-\lambda)^{N-M} \operatorname{det}_{M \times M}\left(\widetilde{\mathcal{L}}\left(\left\{y_{i}\right\}_{M},\left\{x_{i}\right\}_{N}, \omega\right)-\lambda\right) .
$$

The proof is given in the appendix.

We conclude this section by the simplest example of the correspondence between the spectra of Gaudin Hamiltonians and diagonal elements of the CM Lax matrix with fixed eigenvalues. 
The simplest example: the rational $\mathfrak{g} l_{2}$ Gaudin model with 2 marked points (sites) $q_{1,2}= \pm q$ with spins $\frac{1}{2}$ and the twist matrix $v=\left(\begin{array}{cc}\omega & 0 \\ 0 & -\omega\end{array}\right)$. There Hilbert space of the model is 4-dimensional and the states are classified according to eigenvalues of the spin $z$-projection operator:

1) $\left(\begin{array}{l}1 \\ 0\end{array}\right),\left(\begin{array}{l}1 \\ 0\end{array}\right)$. In this case there are no Bethe roots and the spectrum is given by (3.25):

$$
\frac{1}{\hbar} H_{1,2}^{\mathrm{G}}=\omega \pm \frac{\hbar}{2 q}
$$

2) $\left(\begin{array}{l}1 \\ 0\end{array}\right),\left(\begin{array}{l}0 \\ 1\end{array}\right)$ or $\left(\begin{array}{l}0 \\ 1\end{array}\right),\left(\begin{array}{l}1 \\ 0\end{array}\right)$. The single Bethe root satisfies the BE

$$
-2 \omega=\frac{\hbar}{\mu-q}+\frac{\hbar}{\mu+q} .
$$

Substituting its solution(s) $\mu_{ \pm}=-\frac{\hbar}{2 \omega} \pm \frac{\sqrt{4 \omega^{2} q^{2}+\hbar^{2}}}{2 \omega}$ we get the spectrum:

$$
\left.\frac{1}{\hbar} H_{1}^{\mathrm{G}}\right|_{\mu=\mu_{\mp}}= \pm \frac{\sqrt{4 \omega^{2} q^{2}+\hbar^{2}}}{2 q},\left.\quad \frac{1}{\hbar} H_{2}^{\mathrm{G}}\right|_{\mu=\mu_{\mp}}=\mp \frac{\sqrt{4 \omega^{2} q^{2}+\hbar^{2}}}{2 q} .
$$

3) $\left(\begin{array}{l}0 \\ 1\end{array}\right),\left(\begin{array}{l}0 \\ 1\end{array}\right)$. Two Bethe roots satisfy the following BE:

$$
\left\{\begin{array}{l}
-2 \omega+\frac{2 \hbar}{\mu_{1}-\mu_{2}}=\frac{\hbar}{\mu_{1}-q}+\frac{\hbar}{\mu_{1}+q}, \\
-2 \omega+\frac{2 \hbar}{\mu_{2}-\mu_{1}}=\frac{\hbar}{\mu_{2}-q}+\frac{\hbar}{\mu_{2}+q} .
\end{array}\right.
$$

The solutions $\mu_{1}=-\frac{\hbar}{2 \omega} \pm \frac{\sqrt{4 \omega^{2} q^{2}-\hbar^{2}}}{2 \omega}$ and $\mu_{2}=-\frac{\hbar}{2 \omega} \mp \frac{\sqrt{4 \omega^{2} q^{2}-\hbar^{2}}}{2 \omega}$ lead to the spectrum

$$
\frac{1}{\hbar} H_{1,2}^{\mathrm{G}}=-\omega \pm \frac{\hbar}{2 q} .
$$

Let us obtain the same spectrum from the classical rational 2-body CM model with the coupling constant $\hbar$. The Lax matrix is

$$
L^{\mathrm{CM}}=\left(\begin{array}{cc}
\dot{q}_{1} & \frac{\hbar}{q_{1}-q_{2}} \\
-\frac{\hbar}{q_{1}-q_{2}} & \dot{q}_{2}
\end{array}\right)
$$

Let us put $q_{1,2}= \pm q$ as in the Gaudin model. The requirement for this matrix to have eigenvalues $\left(v_{1}, v_{2}\right)$ provides the following values of the velocities:

$$
\dot{q}_{1,2}=\frac{v_{1}+v_{2}}{2} \pm \sqrt{\frac{\left(v_{1}-v_{2}\right)^{2}}{4}+\frac{\hbar^{2}}{4 q^{2}}} .
$$

The QC duality claims that $\dot{q}_{1,2}=\frac{1}{\hbar} H_{1,2}^{\mathrm{G}}$. The above described three cases follow from (4.27) when 1) $\left.\left.v_{1}=v_{2}=\omega, 2\right) v_{1}=\omega, v_{2}=-\omega, 3\right) v_{1}=v_{2}=-\omega$. 


\section{Relation to branes and gauge theories}

In this section we briefly comment on the realization of the QC duality in terms of branes and gauge theories.

First let us note that there are two types of dualities in the integrable systems relevant to our discussion: the bispectrality and the QC duality. The bispectral transformations preserve the class of quantum spin chains. Roughly speaking, the inhomogeneity parameters and twists get interchanged under this transformation. On the classical level, the bispectrality acts by interchanging coordinates and eigenvalues of the Lax operator and preserves the class of CM-RS models of different kinds (rational, trigonometric, elliptic). The mapping can be defined for the classical and quantum models independently with the clear semiclassical picture in between. One can also show that spectral curves (on the classical level) or the systems of $\mathrm{BE}$ (on the quantum level) for the bispectrally dual models are related in a controllable way.

In distinction of the bispectrality, the QC duality we have focused on in this paper is a relation between representatives from the two different families of models: quantum (spin chains, Gaudin) and classical (CM-RS). The spin chain inhomogeneity parameters, twists and Hamiltonians get mapped respectively to coordinates of the CM-RS particles and eigenvalues of the Lax matrix while the Hamiltonians of the spin chain get mapped to velocities of the CM-RS particles. The spectral problem for Hamiltonians of the spin chain (equivalent to solving the $\mathrm{BE}$ ) corresponds to a bit unusual problem at the classical CM-RS side: given values of all integrals of motion in involution, we should fix all coordinates and look for the allowed values of particles velocities (or just momenta in the CM case). In other words, the quantum eigenstates are encoded by intersection points of two Lagrangian submanifolds in the phase space of a classical integrable model.

The interpretation of bispectrality in terms of the gauge theories on the brane worldvolumes in the simplest cases has been found at the CM-RS side in [22] and at the spin chain side in [19]. More recently, the bispectrality transformation has been used to prove the AGT duality $[24,25]$ and the bulk-worldsheet $2 \mathrm{~d} / 4 \mathrm{~d}$ duality for the nonabelian strings [23] in the integrability framework. The comprehensive analysis of the bispectrality for the general case has been developed in [35]. It was identified as the mirror transformation in the quiver $3 \mathrm{~d}$ theory with the generic matter in the fundamental representation. Moreover, the QC duality was interpreted there in the same quiver set-up which encodes the particular brane configuration responsible for the gauge theory [35].

The brane configuration relevant to our quiver gauge theory is as follows. We have $n$ parallel NS5 branes extended in the $\left(x_{0}, x_{1}, x_{2}, x_{7}, x_{8}, x_{9}\right)$ directions, $N_{i}$ D3 branes extended in $\left(x_{0}, x_{1}, x_{2}, x_{3}\right)$ between $i$-th and $(i+1)$-th NS5 branes, and $K_{i}$ D5 branes extended in $\left(x_{0}, x_{1}, x_{2}, x_{3}, x_{4}, x_{5}, x_{6}\right)$ directions between the $i$-th and $(i+1)$-th NS5 branes. From this brane configuration we obtain the $\prod_{i}^{n} \mathrm{U}\left(N_{i}\right)$ gauge group on the D3 brane worldvolume with additional $K_{i}$ fundamentals for the $i$-th gauge group. The distance between the $i$ th and $(i+1)$-th NS5 branes yields the gauge coupling for the $\mathrm{U}\left(N_{i}\right)$ gauge group while coordinates of the D5 branes in the $x_{7}, x_{8}$ plane correspond to the masses of fundamentals. The positions of D3 branes in the $x_{7}, x_{8}$ plane correspond to the coordinates on the Coulomb 
branch in the quiver theory. The additional $\Omega$-deformation reduces the theory with $N=4$ SUSY to the $N=2^{*}$ theory. At the energy scale below the scale dictated by the lengths of the intervals the theory on D3 branes is identified as $N=2^{*} 3 \mathrm{~d}$ quiver gauge theory. In what follows we assume that one coordinate is compact that is the $3 \mathrm{~d}$ theory lives on $R^{2} \times S^{1}$.

The mapping of the gauge theory data into the integrability framework goes as follows. The Yang-Yang function is identified with the twisted superpotential in the $3 \mathrm{~d}$ gauge theory on the D3 branes and its extrema yield solutions to the BE for the $X X Z$ spin chain or equivalently the equation for the supersymmetric vacuum state in the gauge theory $[33,34]$. The D3 branes are identified with the Bethe roots which are distributed according to the ranks of the gauge groups at each of $n$ steps of nesting in $\prod_{i}^{n} \mathrm{U}\left(N_{i}\right)$. Generically, the number of the Bethe roots at different levels of nesting is different. The distances between the NS5 branes define the twists at the different levels of nesting while the positions of the D5 branes in the $x_{7}, x_{8}$ plane correspond to the inhomogeneity parameters of the $X X Z$ spin chain. To complete the dictionary, recall that the anisotropy parameter of the $X X Z$ chain is defined by the radius of the compact dimension while the parameter of the $\Omega$-deformation plays the role of the Planck constant for the $X X Z$ spin chain. To get the $X X X$ chain from the $X X Z$ one, one should just send the radius of the compact coordinate to zero.

In terms of the brane configuration the dualities correspond to particular brane motions. The bispectrality corresponds to the interchange between the Coulomb and Higgs branches that is the mirror symmetry [35]. To this aim, one should adjust the parameters in such a way that two D5 branes become at the same position in the 7,8,9 coordinates. Then one should remove the segment of the D3 brane stretched between two D5 branes and bring it to infinity. The position of the D3 brane in the 7,8,9 coordinates corresponds to the coordinate at the Higgs branch. Under the bispectrality the roles of the D5 and NS5 branes get interchanged and positions of the NS5 branes and D5 branes play the role of inhomogeneity parameters and twists respectively.

The interpretation of the QC duality is more involved [35]. First, we have to perform the Hanany-Witten move and translate all D5 branes to the left. Upon this move we get the configuration involving the $\sum_{i} K_{i}$ D5 branes yielding the left boundary condition, $n$ NS5 branes defining the right boundary condition and $Q$ D3 branes in between, where

$$
Q=\sum_{j=1}^{n} j K_{j} .
$$

Since the distance between boundaries with the Dirichlet and Neumann conditions is large, we get the $N=2^{*} D=4$ gauge theory with the $\mathrm{U}(Q)$ gauge group on $R^{2} \times S^{2} \times I$. The QC duality is now identified as the duality between the $N=2^{*} D=3$ quiver gauge theory with particular content of fundamentals and the $N=2^{*} D=4$ theory with $\mathrm{U}(Q)$ gauge group with nontrivial boundary conditions. The information about the $D=3$ quiver is now encoded in the boundary conditions of the $D=4$ theory via embedding $\mathrm{SU}(2) \rightarrow \mathrm{U}(Q)$ at the left and right boundaries.

Now we are ready to explain the brane interpretation of the QC duality in the degenerate XXX case we have elaborated. At the spin chain side the positions of $n$ NS5 branes 
along the $x_{3}$ direction are identified with the twist parameters $V_{i}$ and the number of the NS5 branes fixes the rank of the group. The positions of the D5 branes along $x_{7}, x_{8}$ are identified with the inhomogeneity parameters $q_{i}$. In the algebraic consideration given above we considered the case when the total number of the $q_{i}$ 's (which is equal to $\sum_{j=1}^{n} j K_{j}$ ) coincides with the number of particles $Q$ at the RS side. To get $Q=N$ we have to put all $N$ D5 branes at the interval between two leftmost NS5 branes as it can be seen from (5.1). In this case $K_{1}=N$ and $K_{j}=0, j=2, \ldots n$.

Upon the Hanany-Witten move we get the $N=2^{*} D=4 \mathrm{U}(N)$ gauge theory on the D3 branes. The object of interest in this theory is the moduli space of the vacua which is known to be parameterized by the $\mathrm{U}(N)$ flat connections on the torus with one marked point with particular holonomy determined by the $\Omega$-deformation parameter [55-57]. This is exactly the description of the phase space of the trigonometric RS model with $N$ particles [58]. One of the radii of this auxiliary torus is the radius of the compact coordinate which the $4 \mathrm{~d}$ theory is defined on. Since we argued above that the reduction from $X X Z$ to $X X X$ implies vanishing of this radius in the $3 \mathrm{~d}$ quiver theory, we have to take the same limit in the $4 \mathrm{~d}$ theory. It can be immediately recognized as the transition from the trigonometric to the rational RS model. Hence we arrive exactly to the duality between the $X X X$ spin chain and the rational RS model as it was discussed in [35].

Now the boundary conditions fix two Lagrangian submanifolds in this space. At the left Dirichlet boundary there are $N$ D5 branes which provide the coordinates for the RS model with $N$ degrees of freedom and correspond to $\mathrm{SU}(N)$ holonomy around the cycle with the vanishing radius. The second $\mathrm{SU}(N)$ holonomy (around the cycle with nonvanishing radius) corresponds to the Neumann boundary conditions imposed by the NS5 branes. Due to the nontrivial monodromy around the marked point two holonomies can not be diagonalized simultaneously and the second one can be identified as the Lax operator of the rational RS model we have discussed above. Hence we arrive at the picture of intersection of two Lagrangian submanifolds. For the trigonometric case, this picture has been discussed for the first time in [49].

Now, the algebraic consideration of the previous section tells us how the positions $V_{i}, i=1 \ldots n$, of the $n$ NS5 branes in the initial quiver 3 d gauge theory (corresponding to the $G L(n)$ spin chain of length $N$ ) provide the multiplicity of the Lax eigenvalues at the RS side. Equation (4.2) tells that $V_{1}$ has multiplicity $N-N_{1}, V_{2}$ multiplicity $N_{2}-N_{1}$ etc. Since $N_{i}$ is just the number of the D3 branes at the $i$-th segment, we could claim that the structure of the clasterization of the Lax eigenvalues in the RS model is dictated by the difference of the D3 branes at the corresponding step of nesting. Hence we obtain a very explicit prescription how the quiver data in the $3 \mathrm{~d}$ theory get mapped into the choice of the particular Lagrangian submanifold in the moduli space of vacua in the $4 \mathrm{~d}$ gauge theory at the interval at small length of interval.

\section{Discussion}

In this paper we have described a clear-cut relationship between the quantum $X X X$ spin chain and the rational classical RS model. This QC duality and its generalization to 
the trigonometric case has been discussed in [35] in the brane framework but an explicit algebraic analysis was missing. We put this on the firm ground and get some important identifications. The spectrum of the $X X X$ spin chain Hamiltonians coincides with possible values of velocities of the RS particles under the conditions that their coordinates equal the inhomogeneity parameters of the spin chain and eigenvalues of the RS Lax matrix coincide with the twist parameters with certain multiplicities depending on the total "spin projection". The stationary states of the quantum model appear to be in one-to-one correspondence with intersection points of two Lagrangian submanifolds in the phase space of the classical model. The brane picture behind this pattern has been presented.

This paper together with [35] has just started the systematic investigation of the new type of dualities in integrable models and their gauge theory meaning. We believe that they may be potentially very useful in many physical applications. In particular, the possibility to find spectra of quantum Hamiltonians in terms of the $Q C$-dual classical model seems to be especially intriguing and promising.

We conclude by a list of some interesting related topics deserving further investigation.

- The generalization of the algebraic analysis to the trigonometric case is straightforward. We expect that the QC duality extends also to integrable models with elliptic $R$-matrices. However, such a generalization is going to be non-trivial since in the elliptic case there are no continuous twist parameters. This probably means that they get quantized.

- It would be extremely interesting to enrich the QC duality by a recipe of finding, via the map to a classical system, not only spectra of quantum Hamiltonians but also the eigenstates themselves. We conjecture that such specifically quantum information might be encoded in the fine structure of the intersection of Lagrangian submanifolds.

- A related problem is to elucidate the meaning of the YY function and Baxter's $Q$ operators in the context of the CM-RS models. The YY function was conjectured [49] to be the generating function for Lagrangian submanifolds in the RS phase space. However, the validity and consequences of this identification deserve further study. As the results of [42] suggest, the Baxter's $Q$-operators should be related to Backlund transformations on the classical side. The details are to be clarified.

- It is important to extend our analysis to the generalized duality suggested in [35] when the number of inhomogeneity parameters at the spin chain side does not coincide with the number of particles at the RS side. In the brane language this corresponds to the generic quiver.

- The quantum-classical duality discussed in this paper should be somehow extended to a quantum-quantum one, when the classical CM or RS model gets quantized. The question is what happens with the spin chains under this deformation. Presumably, they turn into non-stationary models described by equations of the KnizhnikZamolodchikov type. This issue is also closely related to evaluation of knot invariants [59]. 
- So far only two types of the brane moves have been identified as some dualities in the associated integrable systems: the move corresponding to Higgsing and the HananyWitten move. It would be interesting to obtain the dualities corresponding to more general brane moves.

- Recently some new field theory generalizations of higher rank Painlevé-Schlesinger equations and the corresponding models of the Gaudin-Calogero type were suggested [60]. They should respect the same kind of dualities and, therefore, it is tempting to study possible continuous limits of spin chains in order to find the dualities between local and non-local models which may be of special interest.

- As is mentioned in [25], the quantization of the spectral curves of integrable chains leads to the relations (the Baxter equations) which are very similar to their classical analogues. This might allow one to interpret the $\mathrm{QC}$ duality in terms of a combination of the spectral duality $[10,24]$ and the Symplectic Hecke Correspondence [61-67] (cf. [58]).

\section{A Proofs of propositions}

Here we prove Propositions 4.1 and 4.2.

Proposition 4.1. In the appendix we employ the auxiliary notation $\mathbf{x}_{N}=\left\{x_{i}\right\}_{N}, \mathbf{y}_{M}=$ $\left\{y_{i}\right\}_{M}$ for brevity. Another frequently used notation, $\mathbf{e}_{N}$, means the $N$-dimensional vector $(1, \ldots, 1)$, so $\mathbf{x}_{N}-\hbar \mathbf{e}_{N}=\left\{x_{i}-\hbar\right\}_{N}$, etc.

Recall the statement: the pair of matrices

$$
\mathcal{L}_{i j}\left(\mathbf{x}_{N}, \mathbf{y}_{M}, g\right)=\frac{g \hbar}{x_{i}-x_{j}+\hbar} \prod_{k \neq j}^{N} \frac{x_{j}-x_{k}+\hbar}{x_{j}-x_{k}} \prod_{\gamma=1}^{M} \frac{x_{j}-y_{\gamma}}{x_{j}-y_{\gamma}+\hbar}, \quad i, j=1, \ldots, N
$$

and

$$
\widetilde{\mathcal{L}}_{\alpha \beta}\left(\mathbf{y}_{M}, \mathbf{x}_{N}, g\right)=\frac{g \hbar}{y_{\alpha}-y_{\beta}+\hbar} \prod_{\gamma \neq \beta}^{M} \frac{y_{\beta}-y_{\gamma}-\hbar}{y_{\beta}-y_{\gamma}} \prod_{k=1}^{N} \frac{y_{\beta}-x_{k}}{y_{\beta}-x_{k}-\hbar}, \quad \alpha, \beta=1, \ldots, M .
$$

are related by the identity

$$
\operatorname{det}_{N \times N}\left(\mathcal{L}\left(\mathbf{x}_{N}, \mathbf{y}_{M}, g\right)-\lambda\right)=(g-\lambda)^{N-M} \operatorname{det}_{M \times M}\left(\widetilde{\mathcal{L}}\left(\mathbf{y}_{M}, \mathbf{x}_{N}, g\right)-\lambda\right) .
$$

To prove this, we need some technical lemmas.

Lemma A.1. [54] The matrices $\mathcal{L}$ and $\widetilde{\mathcal{L}}$ can be represented in terms of diagonal matrices

$$
\begin{array}{rlrl}
\mathcal{D}_{i j} & =\delta_{i j} \prod_{\gamma=1}^{M} \frac{y_{\gamma}-x_{j}}{y_{\gamma}-x_{j}-\hbar}, & i, j=1, \ldots, N, \\
\widetilde{\mathcal{D}}_{\alpha \beta} & =\delta_{\alpha \beta} \prod_{k=1}^{N} \frac{y_{\beta}-x_{k}}{y_{\beta}-x_{k}-\hbar}, & \alpha, \beta & =1, \ldots, M,
\end{array}
$$


diagonal matrices $D_{0}$ and $D_{\hbar}$

$$
\begin{gathered}
\left(D_{0}\right)_{i j}\left(\mathbf{u}_{K}\right)=\delta_{i j} \prod_{k \neq i}^{K}\left(u_{i}-u_{k}\right), \quad\left(D_{\hbar}\right)_{i j}\left(\mathbf{u}_{K}\right)=\delta_{i j} \prod_{k \neq i}^{K}\left(u_{i}-u_{k}+\hbar\right), \\
i, j=1, \ldots, K
\end{gathered}
$$

the Vandermonde matrix $V_{i j}\left(\mathbf{u}_{K}\right)=u_{j}^{i-1}, i, j=1, \ldots, K$, and the triangular matrix

$$
\left(C_{\hbar, K}\right)_{i j}=\left\{\begin{array}{l}
\frac{(i-1) ! \hbar^{i-j}}{(j-1) !(i-j) !}, j \leq i, \quad i, j=1, \ldots, K \\
0, \quad j>i,
\end{array}\right.
$$

in the following way:

$$
\begin{aligned}
\mathcal{L}\left(\mathbf{x}_{N}, \mathbf{y}_{M}, g\right) & =g D_{\hbar}^{-1}\left(\mathbf{x}_{N}\right) V^{T}\left(\mathbf{x}_{N}+\hbar \mathbf{e}_{N}\right)\left(V^{T}\right)^{-1}\left(\mathbf{x}_{N}\right) D_{\hbar}\left(\mathbf{x}_{N}\right) \mathcal{D} \\
& =g D_{\hbar}^{-1}\left(\mathbf{x}_{N}\right) V^{T}\left(\mathbf{x}_{N}\right) C_{\hbar, N}^{T}\left(V^{T}\right)^{-1}\left(\mathbf{x}_{N}\right) D_{\hbar}\left(\mathbf{x}_{N}\right) \mathcal{D} . \\
\widetilde{\mathcal{L}}\left(\mathbf{y}_{M}, \mathbf{x}_{N}, g\right) & =g D_{0}\left(\mathbf{y}_{M}\right) V^{-1}\left(\mathbf{y}_{M}\right) V\left(\mathbf{y}_{M}-\hbar \mathbf{e}_{M}\right) D_{0}^{-1}\left(\mathbf{y}_{M}\right) \widetilde{\mathcal{D}} \\
& =g D_{0}\left(\mathbf{y}_{M}\right) V^{-1}\left(\mathbf{y}_{M}\right) C_{-\hbar, M} V\left(\mathbf{y}_{M}\right) D_{0}^{-1}\left(\mathbf{y}_{M}\right) \widetilde{\mathcal{D}} .
\end{aligned}
$$

(here $(\ldots)^{T}$ means transposition of the matrix).

Notice that $\operatorname{det} \mathcal{D}=\operatorname{det} \widetilde{\mathcal{D}}$. Therefore, statement (A.3) can be rewritten as

$$
\operatorname{det}_{N \times N}\left(\mathcal{L}_{0}\left(\mathbf{x}_{N}, g\right)-\lambda \mathcal{D}^{-1}\right)=(g-\lambda)^{N-M} \operatorname{det}_{M \times M}\left(\widetilde{\mathcal{L}}_{0}\left(\mathbf{y}_{M}, g\right)-\lambda \widetilde{\mathcal{D}}^{-1}\right),
$$

where

$$
\left(\mathcal{L}_{0}\right)_{i j}\left(\mathbf{x}_{N}, g\right)=\left.\mathcal{L}_{i j}\right|_{M=0}=\frac{g \hbar}{x_{i}-x_{j}+\hbar} \prod_{k \neq j}^{N} \frac{x_{j}-x_{k}+\hbar}{x_{j}-x_{k}}, \quad i, j=1, \ldots, N
$$

and

$$
\left(\widetilde{\mathcal{L}}_{0}\right)_{\alpha \beta}\left(\mathbf{y}_{M}, g\right)=\left.\widetilde{\mathcal{L}}_{i j}\right|_{N=0}=\frac{g \hbar}{y_{\alpha}-y_{\beta}+\hbar} \prod_{\gamma \neq \beta}^{M} \frac{y_{\beta}-y_{\gamma}-\hbar}{y_{\beta}-y_{\gamma}}, \quad \alpha, \beta=1, \ldots, M .
$$

Lemma A.2. The l.h.s. of (A.3) (or that of (A.9)), i.e., the function

$$
\left|\mathcal{L}_{N}\right|(M) \stackrel{\text { def }}{=} \operatorname{det}_{N \times N}\left(\mathcal{L}_{0}\left(\mathbf{x}_{N}, g\right)-\lambda \mathcal{D}^{-1}\right)
$$

has no poles of the form $\frac{1}{x_{a}-x_{b}}$ or $\frac{1}{x_{a}-x_{b}+\hbar}$ for all $a, b=1, \ldots, N$. All poles of (A.12) come from the diagonal matrix $\mathcal{D}^{-1}$.

Proof. The idea is to represent $\left|\mathcal{L}_{N}\right|(M)$ in the form of determinant of a matrix whose elements have no poles of the form $\frac{1}{x_{a}-x_{b}}$ or $\frac{1}{x_{a}-x_{b}+\hbar}$ for all $a, b=1, \ldots, N$. Using Lemma A.1 we have

$$
\begin{aligned}
\operatorname{det}\left(\mathcal{L}_{0}-\lambda \mathcal{D}^{-1}\right) & =\operatorname{det}\left(\mathcal{L}_{0}^{T}-\lambda \mathcal{D}^{-1}\right) \\
& =\operatorname{det}\left(g D_{\hbar} V^{-1} C_{\hbar, N} V D_{\hbar}^{-1}-\lambda \mathcal{D}^{-1}\right)=\operatorname{det}\left(g C_{\hbar, N}-\lambda V \mathcal{D}^{-1} V^{-1}\right) .
\end{aligned}
$$


The latter expression does not contain any poles of the type $\frac{1}{x_{a}-x_{b}+\hbar}$. However, it may contain poles of the type $\frac{1}{x_{a}-x_{b}}$ since $\operatorname{det} V=\prod_{i>j}\left(x_{i}-x_{j}\right)$. Let us verify that all such poles vanish if $\mathcal{D}$ is given by (A.4). Indeed, the inverse of the Vandermonde matrix is given by

$$
V_{k j}^{-1}=\left.\frac{1}{(j-1) !} \partial_{\rho}^{(j-1)} \prod_{s \neq k}^{N} \frac{\rho-x_{s}}{x_{k}-x_{s}}\right|_{\rho=0} .
$$

Therefore, the matrix element $\left(V \mathcal{D}^{-1} V^{-1}\right)_{i j}$ takes the form

$$
\left(V \mathcal{D}^{-1} V^{-1}\right)_{i j}=\sum_{k=1}^{N} V_{i k} \mathcal{D}_{k k}^{-1} V_{k j}^{-1}=\left.\sum_{k=1}^{N} x_{k}^{i-1} \mathcal{D}_{k k}^{-1} \frac{1}{(j-1) !} \partial_{\rho}^{(j-1)} \prod_{s \neq k}^{N} \frac{\rho-x_{s}}{x_{k}-x_{s}}\right|_{\rho=0} .
$$

Consider the linear combination of columns $\sum_{k=1}^{N} x_{k}^{i-1} \mathcal{D}_{k k}^{-1} \prod_{s \neq k}^{N} \frac{\rho-x_{s}}{x_{k}-x_{s}}=\sum_{k=1}^{N}\left(V \mathcal{D}^{-1} V^{-1}\right)_{i k} \rho^{k-1}$ which is a generating function for them. Powers of the auxiliary variable $\rho$ correspond to the values of $j-1=0, \ldots, N-1$. An arbitrary pole $\frac{1}{x_{a}-x_{b}}$ appears in the sum for $k=a, b$. The residue is given by

$$
\prod_{s=1}^{N}\left(\rho-x_{s}\right)\left(\frac{x_{a}^{i-1}}{\rho-x_{a}} \frac{\mathcal{D}_{a a}^{-1}}{\prod_{l \neq a, b}\left(x_{a}-x_{l}\right)}-\frac{x_{b}^{i-1}}{\rho-x_{b}} \frac{\mathcal{D}_{b b}^{-1}}{\prod_{l \neq a, b}\left(x_{b}-x_{l}\right)}\right) .
$$

This expression vanishes at $x_{a}=x_{b}$ if $\mathcal{D}_{a a}^{-1}=\mathcal{D}_{a a}^{-1}\left(x_{a}\right)$. This is the case of (A.4).

Lemma A.3. The r.h.s. of (A.3) (or that of (A.9)), i.e. the function

$$
\left|\widetilde{\mathcal{L}}_{M}\right|(N) \stackrel{\text { def }}{=} \operatorname{det}_{M \times M}\left(\widetilde{\mathcal{L}}_{0}\left(\mathbf{y}_{M}, g\right)-\lambda \widetilde{\mathcal{D}}^{-1}\right)
$$

has no poles of the form $\frac{1}{y_{a}-y_{b}}$ or $\frac{1}{y_{a}-y_{b}+\hbar}$ for all $a, b=1, \ldots, M$. All poles of (A.17) come from the diagonal matrix $\widetilde{\mathcal{D}}$.

The proof is similar to the previous Lemma A.2.

Proof of Proposition 4.1. The proof is by induction in $M$. The nontrivial part of $C_{\hbar, K}$ has a form of the left-justified Pascal's triangle (of binomial coefficients) weighted by $\hbar^{i-j}$. Notice that $\left(C_{\hbar, K}\right)_{j j}=1$ for all $j=1, \ldots, K$. Therefore,

$$
\operatorname{det}\left(g C_{\hbar, K}-\lambda\right)=(g-\lambda)^{K} .
$$

Let us first check (A.3) for $M=0$ and arbitrary $N$ (or $N=0$ and arbitrary $M$ ). Since $\left.\mathcal{D}\right|_{M=0}=\operatorname{Id}_{N}$, it follows from (A.8) that

$$
\operatorname{det}\left(\mathcal{L}_{0}-\lambda\right)=\operatorname{det}\left(g C_{\hbar, N}-\lambda\right)=(g-\lambda)^{N} .
$$

Similarly, $\operatorname{det}\left(\widetilde{\mathcal{L}}_{0}-\lambda\right)=(g-\lambda)^{M}$. Suppose (the induction assumption) that (A.9) holds true for all $N$ and some fixed $M-1$, i.e.,

$$
\left|\mathcal{L}_{N}\right|(M-1)=(g-\lambda)^{N-M+1}\left|\widetilde{\mathcal{L}}_{M-1}\right|(N), \quad \forall N
$$

In order to prove that (A.9) holds for $M-1 \rightarrow M$, we expand both sides of (A.9) as sums over poles in $y_{M}$ and compare the results. 
1) Consider first the 1.h.s. of (A.9):

$$
\left|\mathcal{L}_{N}\right|(M)=\operatorname{det}\left\|\frac{g \hbar}{x_{i}-x_{j}+\hbar} \prod_{k \neq j}^{N} \frac{x_{j}-x_{k}+\hbar}{x_{j}-x_{k}}-\lambda \delta_{i j} \prod_{\gamma=1}^{M} \frac{y_{\gamma}-x_{j}-\hbar}{y_{\gamma}-x_{j}}\right\|,
$$

where $i, j=1, \ldots, N$. Notice that $\left|\mathcal{L}_{N}\right|(M)$ is a rational function of the $y_{M}$ with simple poles at $x_{1}, \ldots, x_{N}$. Therefore, it can be represented in the form

$$
\left|\mathcal{L}_{N}\right|(M)=\left.\left|\mathcal{L}_{N}\right|(M)\right|_{y_{M}=\infty}+\sum_{l=1}^{N} \frac{1}{y_{M}-x_{l}} C_{l} .
$$

The first term equals $\mathcal{L}_{N}|(M)|_{y_{M}=\infty}=\left|\mathcal{L}_{N}\right|(M-1)$. To find $C_{l}$, let us note that the pole $\frac{1}{y_{M}-x_{l}}$ appears only in the $l l$-th component of the second (diagonal) term of matrix (A.21), $-\lambda \mathcal{D}_{l l}^{-1}$. Hence,

$$
C_{l}=\left.\Delta_{l l}\right|_{y_{M}=x_{l}} \operatorname{Res}_{y_{M}=x_{l}}\left(-\lambda \mathcal{D}_{l l}^{-1}\right),
$$

where $\Delta_{l l}$ is the principal minor of the matrix $\mathcal{L}_{N}$ obtained by removing the $l$-th column and the $l$-th row. It is easy to see that

$$
\left.\Delta_{l l}\right|_{y_{M}=x_{l}}=\left|\mathcal{L}_{N-1}^{l}\right|(M-1) \prod_{j \neq l}^{N} \frac{x_{j}-x_{l}+\hbar}{x_{j}-x_{l}},
$$

where the index $l$ in $\mathcal{L}_{N-1}^{l}$ emphasizes that its argument is $\mathbf{x}_{N-1}=$ $x_{1}, \ldots, x_{l-1}, x_{l+1}, \ldots, x_{N}$, i.e. $\left\{\mathbf{x}_{N}\right\} \backslash x_{l}$. The residue in (A.23) equals $\hbar \lambda \prod_{\gamma=1}^{M-1} \frac{y_{\gamma}-x_{j}-\hbar}{y_{\gamma}-x_{j}}$. Then expression (A.22) takes the form

$$
\begin{aligned}
\left|\mathcal{L}_{N}\right|(M)=\left|\mathcal{L}_{N}\right|(M-1) & +\sum_{l=1}^{N} \frac{\hbar \lambda}{y_{M}-x_{l}} \times \\
& \times \prod_{\gamma=1}^{M-1} \frac{y_{\gamma}-x_{j}-\hbar}{y_{\gamma}-x_{j}}\left|\mathcal{L}_{N-1}^{l}\right|(M-1) \prod_{j \neq l}^{N} \frac{x_{j}-x_{l}+\hbar}{x_{j}-x_{l}} .
\end{aligned}
$$

By the induction assumption, the determinants $\left|\mathcal{L}_{N}\right|(M-1)$ and $\left|\mathcal{L}_{N-1}^{l}\right|(M-1)$, $l=1, \ldots, N$ satisfy (A.9), i.e.,

$$
\begin{aligned}
\left|\mathcal{L}_{N}\right|(M-1) & =(g-\lambda)^{N-M+1}\left|\widetilde{\mathcal{L}}_{M-1}\right|(N), \\
\left|\mathcal{L}_{N-1}^{l}\right|(M-1) & =(g-\lambda)^{N-M}\left|\widetilde{\mathcal{L}}_{M-1}\right|(N-1)_{l}
\end{aligned}
$$

The lower index $l$ in the r.h.s. again emphasizes that the set of its arguments is $\mathbf{x}_{N-1}=\left\{\mathbf{x}_{N}\right\} \backslash x_{l}$.

2) The r.h.s. of (A.9) is determined by

$$
\left|\widetilde{\mathcal{L}}_{M}\right|(N)=\operatorname{det}\left\|\frac{g \hbar}{y_{\alpha}-y_{\beta}+\hbar} \prod_{\gamma \neq \beta}^{M} \frac{y_{\beta}-y_{\gamma}-\hbar}{y_{\beta}-y_{\gamma}}-\lambda \delta_{\alpha \beta} \prod_{k=1}^{N} \frac{y_{\beta}-x_{k}-\hbar}{y_{\beta}-x_{k}}\right\|
$$


where $\alpha, \beta=1, \ldots, M$. As it follows from Lemma A.3, $\left|\widetilde{\mathcal{L}}_{M}\right|(N)$ has no poles of the type $\frac{1}{y_{a}-y_{b}}$ or $\frac{1}{y_{a}-y_{b}+\hbar}$. Therefore, similarly to (A.22), we have the decomposition

$$
\left|\widetilde{\mathcal{L}}_{M}\right|(N)=\left.\left|\widetilde{\mathcal{L}}_{M}\right|(N)\right|_{y_{M}=\infty}+\sum_{l=1}^{N} \frac{1}{y_{M}-x_{l}} \tilde{C}_{l} .
$$

At $y_{M}=\infty$ the matrix $\widetilde{\mathcal{L}}_{M}$ takes the form $\left(\begin{array}{cc}\widetilde{\mathcal{L}}_{M-1} & 0 \\ 0 & g-\lambda\end{array}\right)$. Hence,

$$
\left.\left|\widetilde{\mathcal{L}}_{M}\right|(N)\right|_{y_{M}=\infty}=(g-\lambda)\left|\widetilde{\mathcal{L}}_{M-1}\right|(N) .
$$

To find $\tilde{C}_{l}$, notice that all poles of the type $\frac{1}{y_{M}-x_{l}}, l=1, \ldots, N$, are contained only in the element $\left(\widetilde{\mathcal{L}}_{M}\right)_{M M}$. Therefore, $\tilde{C}_{l}=\left.\Delta_{M M}\right|_{y_{M}=x_{l}} \underset{y_{M}=x_{l}}{\operatorname{Res}}\left(\widetilde{\mathcal{L}}_{M}\right)_{M M}$. It is easy to see that $\operatorname{Res}_{y_{M}=x_{l}}\left(\widetilde{\mathcal{L}}_{M}\right)_{M M}=\lambda \hbar \prod_{k \neq l}^{N} \frac{x_{l}-x_{k}-\hbar}{x_{l}-x_{k}}$ and

$$
\left.\Delta_{M M}\right|_{y_{M}=x_{l}}=\left|\widetilde{\mathcal{L}}_{M-1}\right|(N-1)_{l} \prod_{\beta=1}^{M-1} \frac{y_{\beta}-x_{l}-\hbar}{y_{\beta}-x_{l}},
$$

where $\left|\widetilde{\mathcal{L}}_{M-1}\right|(N-1)_{l}$ is defined in (A.26). Finally, for (A.28) we have

$$
\begin{aligned}
\left|\widetilde{\mathcal{L}}_{M}\right|(N)=(g-\lambda)\left|\widetilde{\mathcal{L}}_{M-1}\right|(N)+\sum_{l=1}^{N} \frac{\lambda \hbar}{y_{M}-x_{l}} \times & \\
\times & \prod_{k \neq l}^{N} \frac{x_{l}-x_{k}-\hbar}{x_{l}-x_{k}}\left|\widetilde{\mathcal{L}}_{M-1}\right|(N-1)_{l} \prod_{\beta=1}^{M-1} \frac{y_{\beta}-x_{l}-\hbar}{y_{\beta}-x_{l}} .
\end{aligned}
$$

3) At last, compare (A.25) with (A.26) and (A.31). In this way we arrive at the equality

$$
\left|\mathcal{L}_{N}\right|(M)=(g-\lambda)^{N-M}\left|\widetilde{\mathcal{L}}_{M}\right|(N), \quad \forall N
$$

that finishes the proof.

Proposition 4.2: the "non-relativistic" limit $\hbar \rightarrow \mathbf{0}$. The analogue of Lemma A.1 is

Lemma A.4. [54] The matrices

$$
\begin{gathered}
\mathcal{L}_{i j}\left(\mathbf{x}_{N}, \mathbf{y}_{M}, \omega\right)=\delta_{i j}\left(\omega+\sum_{k \neq i}^{N} \frac{\hbar}{q_{i}-q_{k}}+\sum_{\gamma=1}^{M} \frac{\hbar}{\mu_{\gamma}-q_{i}}\right)+\left(1-\delta_{i j}\right) \frac{\hbar}{q_{i}-q_{j}}, \\
i, j=1, \ldots, N
\end{gathered}
$$

and

$$
\begin{gathered}
\widetilde{\mathcal{L}}_{\alpha \beta}\left(\mathbf{y}_{M}, \mathbf{x}_{N}, \omega\right)=\delta_{\alpha \beta}\left(\omega-\sum_{\gamma \neq \alpha}^{M} \frac{\hbar}{\mu_{\alpha}-\mu_{\gamma}}-\sum_{k=1}^{N} \frac{\hbar}{q_{k}-\mu_{\alpha}}\right)+\left(1-\delta_{\alpha \beta}\right) \frac{\hbar}{\mu_{\alpha}-\mu_{\beta}}, \\
\alpha, \beta=1, \ldots, M .
\end{gathered}
$$


can be represented in terms of the diagonal matrices

$$
\begin{aligned}
\mathcal{D}_{i j}=\delta_{i j} \sum_{\gamma=1}^{M} \frac{\hbar}{\mu_{\gamma}-q_{i}}, & i, j=1, \ldots, N, \\
\widetilde{\mathcal{D}}_{\alpha \beta}=\delta_{\alpha \beta} \sum_{k=1}^{N} \frac{\hbar}{\mu_{\alpha}-q_{k}}, & \alpha, \beta=1, \ldots, M,
\end{aligned}
$$

the diagonal matrix

$$
\left(D_{0}\right)_{i j}\left(\mathbf{u}_{K}\right)=\delta_{i j} \prod_{k \neq i}^{K}\left(u_{i}-u_{k}\right), \quad i, j=1, \ldots, K
$$

the Vandermonde matrix

$$
V_{i j}\left(\mathbf{u}_{K}\right)=u_{j}^{i-1}, \quad i, j=1, \ldots, K
$$

and

$$
\left(C_{0, K}\right)_{i j}= \begin{cases}j, & i=j+1, i=2, \ldots, K, \\ 0, & \text { otherwise }\end{cases}
$$

in the following way:

$$
\begin{aligned}
\mathcal{L}\left(\mathbf{x}_{N}, \mathbf{y}_{M}, \omega\right) & =\omega+\hbar D_{0}^{-1}\left(\mathbf{x}_{N}\right) \partial_{z} V^{T}\left(\mathbf{x}_{N}+z \mathbf{e}_{N}\right)\left(V^{T}\right)^{-1}\left(\mathbf{x}_{N}+z \mathbf{e}_{N}\right) D_{0}\left(\mathbf{x}_{N}\right)+\mathcal{D} \\
& =\omega+\hbar D_{0}^{-1}\left(\mathbf{x}_{N}\right) V^{T}\left(\mathbf{x}_{N}\right) C_{0, N}^{T}\left(V^{T}\right)^{-1}\left(\mathbf{x}_{N}\right) D_{0}\left(\mathbf{x}_{N}\right)+\mathcal{D} \\
\widetilde{\mathcal{L}}_{\alpha \beta}\left(\mathbf{y}_{M}, \mathbf{x}_{N}, \omega\right) & =\omega+\hbar D_{0}\left(\mathbf{y}_{M}\right) V^{-1}\left(\mathbf{y}_{M}-z \mathbf{e}_{M}\right) \partial_{z} V\left(\mathbf{y}_{M}-z \mathbf{e}_{M}\right) D_{0}^{-1}\left(\mathbf{y}_{M}\right)+\widetilde{\mathcal{D}} \\
& =\omega-\hbar D\left(\mathbf{y}_{M}\right) V^{-1}\left(\mathbf{y}_{M}\right) C_{0, M} V\left(\mathbf{y}_{M}\right) D^{-1}\left(\mathbf{y}_{M}\right)+\widetilde{\mathcal{D}}
\end{aligned}
$$

Proposition 4.2 can be proved either directly or by taking the limit $\hbar \rightarrow 0$ together with the substitutions $g:=\exp (\hbar \omega), \quad \lambda:=\exp (\hbar \lambda)$. After taking the limit one should also rescale the variables as

$$
x_{i} \rightarrow x_{i} / \hbar, \quad i=1, \ldots, N, y_{\gamma} \rightarrow y_{\gamma} / \hbar, \quad \gamma=1, \ldots, M
$$

Then the statement of Proposition 4.2 (4.21) with the matrices (4.19), (4.20) follows from Proposition 4.1 (4.6) for the matrices (4.4), (4.5). The relation between (4.4), (4.5) and (4.19), (4.20) is given by (2.7) with $\eta=\hbar$. The matrices $C_{\hbar, K}$ and $C_{0, K}$ are related in the same way.

Open Access. This article is distributed under the terms of the Creative Commons Attribution License (CC-BY 4.0), which permits any use, distribution and reproduction in any medium, provided the original author(s) and source are credited. 


\section{References}

[1] N. Seiberg and E. Witten, Electric-magnetic duality, monopole condensation and confinement in $N=2$ supersymmetric Yang-Mills theory, Nucl. Phys. B 426 (1994) 19 [Erratum ibid. B 430 (1994) 485-486] [hep-th/9407087] [INSPIRE].

[2] N. Seiberg and E. Witten, Monopoles, duality and chiral symmetry breaking in $N=2$ supersymmetric QCD, Nucl. Phys. B 431 (1994) 484 [hep-th/9408099] [INSPIRE].

[3] A. Gorsky, I. Krichever, A. Marshakov, A. Mironov and A. Morozov, Integrability and Seiberg-Witten exact solution, Phys. Lett. B 355 (1995) 466 [hep-th/9505035] [INSPIRE].

[4] R. Donagi and E. Witten, Supersymmetric Yang-Mills theory and integrable systems, Nucl. Phys. B 460 (1996) 299 [hep-th/9510101] [INSPIRE].

[5] A. Gorsky and A. Mironov, Integrable many body systems and gauge theories, hep-th/0011197 [INSPIRE].

[6] M. Adams, J.P. Harnad and J. Hurtubise, Dual moment maps into loop algebras, Lett. Math. Phys. 20 (1990) 299 [inSPIRE].

[7] J.P. Harnad, Dual isomonodromic deformations and moment maps to loop algebras, Commun. Math. Phys. 166 (1994) 337 [hep-th/9301076] [INSPIRE].

[8] E. Mukhin, V. Tarasov and A. Varchenko, Bispectral and $\left(g l_{N}, g l_{M}\right)$ dualities, math.QA/0510364.

[9] E. Mukhin, V. Tarasov and A. Varchenko, Bispectral and $\left(g l_{N}, g l_{M}\right)$ dualities, discrete versus differential, math.QA/0605172.

[10] A. Mironov, A. Morozov, B. Runov, Y. Zenkevich and A. Zotov, Spectral duality between Heisenberg chain and Gaudin model, Lett. Math. Phys. 103 (2013) 299 [arXiv:1206.6349] [INSPIRE].

[11] M. Gaudin, Modèles exacts en mècanique statistique: la mèthode de Bethe et ses gènèralizations, Note CEA 15591 and 2 (1972).

[12] M. Gaudin, Diagonalisation dùne classe d'hamiltoniens de spin, J. de Physique 37 (1976) 1087.

[13] F. Calogero, Exactly solvable one-dimensional many body problems, Lett. Nuovo Cim. 13 (1975) 411 [InSPIRE].

[14] F. Calogero, On a functional equation connected with integrable many body problems, Lett. Nuovo Cim. 16 (1976) 77 [InSPIRE].

[15] J. Moser, Three integrable Hamiltonian systems connected with isospectral deformations, Adv. Math. 16 (1975) 1.

[16] M. Olshanetsky and A. Perelomov, Classical integrable finite dimensional systems related to Lie algebras, Phys. Rept. 71 (1981) 313 [InSPIRE].

[17] S.N.M. Ruijsenaars and H. Schneider, A new class of integrable systems and its relation to solitons, Ann. Phys. 146 (1986) 1.

[18] S.N.M. Ruijsenaars, Complete integrability of relativistic Calogero-Moser systems and elliptic function identities, Commun. Math. Phys. 110 (1987) 191. 
[19] A. Gorsky, S. Gukov and A. Mironov, Multiscale $N=2$ SUSY field theories, integrable systems and their stringy/brane origin. 1, Nucl. Phys. B 517 (1998) 409 [hep-th/9707120] [INSPIRE].

[20] H. Braden, A. Marshakov, A. Mironov and A. Morozov, On double elliptic integrable systems. 1. A duality argument for the case of SU(2), Nucl. Phys. B 573 (2000) 553 [hep-th/9906240] [INSPIRE].

[21] S. Ruijsenaars, Action angle maps and scattering theory for some finite dimensional integrable systems. 1. The pure soliton case, Commun. Math. Phys. 115 (1988) 127 [INSPIRE].

[22] V. Fock, A. Gorsky, N. Nekrasov and V. Rubtsov, Duality in integrable systems and gauge theories, JHEP 07 (2000) 028 [hep-th/9906235] [INSPIRE].

[23] K. Bulycheva, H.-Y. Chen, A. Gorsky and P. Koroteev, BPS states in omega background and integrability, JHEP 10 (2012) 116 [arXiv:1207.0460] [INSPIRE].

[24] A. Mironov, A. Morozov, Y. Zenkevich and A. Zotov, Spectral duality in integrable systems from AGT conjecture, JETP Lett. 97 (2013) 45 [arXiv: 1204.0913] [INSPIRE].

[25] A. Mironov, A. Morozov, B. Runov, Y. Zenkevich and A. Zotov, Spectral dualities in XXZ spin chains and five dimensional gauge theories, JHEP 12 (2013) 034 [arXiv:1307.1502] [INSPIRE].

[26] V.V. Mangazeev, V.V. Bazhanov and S.M. Sergeev, An integrable 3D lattice model with positive Boltzmann weights, J. Phys. A 46 (2013) 465206 [arXiv:1308.4773] [INSPIRE].

[27] A. Mironov, A. Morozov and S. Shakirov, Towards a proof of AGT conjecture by methods of matrix models, Int. J. Mod. Phys. A 27 (2012) 1230001 [arXiv:1011.5629] [InSPIRE].

[28] D. Gaiotto, $N=2$ dualities, JHEP 08 (2012) 034 [arXiv:0904.2715] [INSPIRE].

[29] L.F. Alday, D. Gaiotto and Y. Tachikawa, Liouville correlation functions from four-dimensional gauge theories, Lett. Math. Phys. 91 (2010) 167 [arXiv:0906.3219] [INSPIRE].

[30] N. Wyllard, $A(N-1)$ conformal Toda field theory correlation functions from conformal $N=2 \mathrm{SU}(N)$ quiver gauge theories, JHEP 11 (2009) 002 [arXiv:0907.2189] [INSPIRE].

[31] A. Mironov and A. Morozov, On AGT relation in the case of $U(3)$, Nucl. Phys. B 825 (2010) 1 [arXiv:0908.2569] [INSPIRE].

[32] A. Mironov and A. Morozov, Nekrasov functions and exact Bohr-Zommerfeld integrals, JHEP 04 (2010) 040 [arXiv: 0910.5670] [INSPIRE].

[33] N.A. Nekrasov and S.L. Shatashvili, Supersymmetric vacua and Bethe ansatz, Nucl. Phys. Proc. Suppl. 192-193 (2009) 91 [arXiv:0901.4744] [INSPIRE].

[34] N.A. Nekrasov and S.L. Shatashvili, Quantization of integrable systems and four dimensional gauge theories, arXiv:0908.4052 [INSPIRE].

[35] D. Gaiotto and P. Koroteev, On three dimensional quiver gauge theories and integrability, JHEP 05 (2013) 126 [arXiv: 1304.0779] [INSPIRE].

[36] A. Givental and B.-s. Kim, Quantum cohomology of flag manifolds and Toda lattices, Commun. Math. Phys. 168 (1995) 609 [hep-th/9312096] [INSPIRE]. 
[37] E. Mukhin, V. Tarasov and A. Varchenko, Gaudin hamiltonians generate the Bethe algebra of a tensor power of vector representation of $g l_{N}$, St. Petersburg Math. J. 22 (2011) 463 [arXiv: 0904.2131].

[38] E. Mukhin, V. Tarasov and A. Varchenko, KZ characteristic variety as the zero set of classical Calogero-Moser hamiltonians, SIGMA 8 (2012) 72 [arXiv:1201.3990].

[39] A. Alexandrov, V. Kazakov, S. Leurent, Z. Tsuboi and A. Zabrodin, Classical tau-function for quantum spin chains, JHEP 09 (2013) 064 [arXiv:1112.3310] [INSPIRE].

[40] A. Zabrodin, The master T-operator for vertex models with trigonometric R-matrices as classical tau-function, Teor. Mat. Fys. 171 (2013) 59 [Theor. Math. Phys. 174 (2013) 52] [arXiv: 1205.4152] [INSPIRE].

[41] A. Alexandrov, S. Leurent, Z. Tsuboi and A. Zabrodin, The master T-operator for the Gaudin model and the KP hierarchy, arXiv:1306.1111 [INSPIRE].

[42] I. Krichever, O. Lipan, P. Wiegmann and A. Zabrodin, Quantum integrable systems and elliptic solutions of classical discrete nonlinear equations, Commun. Math. Phys. 188 (1997) 267 [hep-th/9604080] [INSPIRE].

[43] L. Faddeev, E. Sklyanin and L. Takhtajan, The quantum inverse problem method. 1, Theor. Math. Phys. 40 (1980) 688 [INSPIRE].

[44] L. Takhtajan and L. Faddeev, The quantum method of the inverse problem and the Heisenberg XYZ model, Russ. Math. Surveys 34 (1979) 11 [INSPIRE].

[45] L. Faddeev, How algebraic Bethe ansatz works for integrable model, hep-th/9605187 [INSPIRE].

[46] V.E. Korepin, N.M. Bogoliubov and A.G. Izergin, Quantum inverse scattering method and correlation functions, Cambridge Monographs on Mathematical Physics, Cambride University Press, Cambridge U.K. (1997).

[47] M. Gaudin, La fonction d'onde de Bethe, Masson, Paris, France (1983).

[48] N.A. Slavnov, The algebraic Bethe ansatz and quantum integrable systems, Russ. Math. Surv. 62 (2007) 727.

[49] N. Nekrasov, A. Rosly and S. Shatashvili, Darboux coordinates, Yang-Yang functional and gauge theory, Nucl. Phys. Proc. Suppl. 216 (2011) 69 [arXiv:1103.3919] [InSPIRE].

[50] P.P. Kulish and N.Yu. Reshetikhin, Diagonalisation of $g l_{N}$ invariant transfer matrices and quantum N-wave system (Lee model), J. Phys. A 16 (1983) L591.

[51] E. Mukhin, V. Tarasov and A. Varchenko, Bethe eigenvectors of higher transfer matrices, J. Stat. Mech. Theory Exp. 8 (2006) 2 [math.QA/0605015].

[52] S. Belliard and É. Ragoucy, Nested Bethe ansatz for 'all' closed spin chains, J. Phys. A 41 (2008) 295202 [arXiv:0804.2822] [InSPIRE].

[53] A. Arnaudon, N. Crampé, A. Doikou, L. Frappat and É. Ragoucy, Analytical Bethe Ansatz for closed and open $g l_{n}$-spin chains in any representation, J. Stat. Mech. 0502 (2005) P02007 [math-ph/0411021] [INSPIRE].

[54] G. Aminov, S. Arthamonov, A. Smirnov and A. Zotov, Modifications of bundles as generating functions of Lax operators, Preprint ITEP-TH 27/13.

[55] N. Nekrasov and E. Witten, The omega deformation, branes, integrability and Liouville theory, JHEP 09 (2010) 092 [arXiv: 1002.0888] [INSPIRE]. 
[56] D. Gaiotto and E. Witten, S-duality of boundary conditions in $N=4$ super Yang-Mills theory, Adv. Theor. Math. Phys. 13 (2009) 721 [arXiv:0807.3720] [InSPIRE].

[57] D. Gaiotto and E. Witten, Supersymmetric boundary conditions in $N=4$ super Yang-Mills theory, J. Statist. Phys. 135 (2009) 789 [arXiv:0804.2902] [INSPIRE].

[58] A. Gorsky and N. Nekrasov, Relativistic Calogero-Moser model as gauged WZW theory, Nucl. Phys. B 436 (1995) 582 [hep-th/9401017] [INSPIRE].

[59] K. Bulycheva and A. Gorsky BPS states in $\Omega$-background and torus knots invariants, to appear.

[60] G. Aminov, S. Arthamonov, A. Levin, M. Olshanetsky and A. Zotov, Painlevé field theory, arXiv: 1306.3265 [INSPIRE].

[61] A.M. Levin, M.A. Olshanetsky and A. Zotov, Hitchin systems - Symplectic Hecke correspondence and two-dimensional version, Commun. Math. Phys. 236 (2003) 93 [nlin/0110045].

[62] A. Zotov and A. Levin, Integrable model of interacting elliptic tops, Theor. Math. Phys. 146 (2006) 45 [INSPIRE].

[63] A. Levin, M. Olshanetsky, A. Smirnov and A. Zotov, Characteristic classes and integrable systems. General construction, Commun. Math. Phys. 316 (2012) 1 [arXiv:1006.0702] [INSPIRE].

[64] A. Levin, M. Olshanetsky, A. Smirnov and A. Zotov, Characteristic classes and integrable systems for simple Lie groups, J. Geom. Phys. 62 (2012) 1810 [arXiv:1007.4127] [InSPIRE].

[65] A. Levin, M. Olshanetsky, A. Smirnov and A. Zotov, Hecke transformations of conformal blocks in WZW theory. I. KZB equations for non-trivial bundles, SIGMA 8 (2012) 095 [arXiv: 1207.4386] [INSPIRE].

[66] A. Levin, M. Olshanetsky, A. Smirnov and A. Zotov, Characteristic classes of SL(N)-bundles and quantum dynamical elliptic R-matrices, J. Phys. A 46 (2013) 035201 [arXiv:1208.5750] [INSPIRE].

[67] A. Levin, M. Olshanetsky and A. Zotov, Painleve VI, rigid tops and reflection equation, Commun. Math. Phys. 268 (2006) 67 [math/0508058] [INSPIRE]. 